\title{
Particle Generation to Minimize the Computing Time of the Discrete Element Method for Particle Packing Simulation
}

\author{
Jinsu Nam \\ Kumoh National Institute of Technology \\ Jaehee Lyu \\ Kumoh National Institute of Technology \\ Junyoung Park ( $\square$ pcello@kumoh.ac.kr) \\ Kumoh National Institute of Technology
}

\section{Research Article}

Keywords: Particle generation, Powder packing, Growing particle, Discrete Element Method

Posted Date: August 31st, 2021

DOI: https://doi.org/10.21203/rs.3.rs-844878/v1

License: (c) (i) This work is licensed under a Creative Commons Attribution 4.0 International License.

Read Full License 
Particle generation to minimize the computing time of the discrete element method for particle packing simulation

Jinsu Nam ${ }^{\mathrm{a}}$, Jaehee Lyu ${ }^{\mathrm{a}}$, Junyoung Park ${ }^{\mathrm{a}, \mathrm{b}, *}$

a Department of Mechanical Design Engineering, Kumoh National Institute of Technology, 61 Daehak-Ro, Gumi, Gyeungbuk 39177, Korea

${ }^{\mathrm{b}}$ Department of Aeronautic, Mechanical and Electrical Convergence Engineering, Kumoh National Institute of Technology, 61, Daehak-Ro, Gumi, Gyeungbuk 39177, Korea

*Corresponding author email: pcello@kumoh.ac.kr

\begin{abstract}
There are computation time constraints caused by the number and size of particles in the powder packing simulation using DEM. In this paper, newly suggested packing model transforms a general packing sequence - particle generation, stack, and compression - into particle generation and packing by growing particles. To verify the new packing model, it was compared using three contact models widely used in DEM, in terms of Radial Distribution Function, porosity, and Coordination Number. As a result, contact between particles showed a similar trend, and the pore distribution was also similar. Using the new packing model can reduce simulation time by $400 \%$ compared to the normal packing model without any other coarse graining methods. This model has only been applied to particle packing simulations in this paper, but it can be expanded to other simulations with complex domain based on DEM.
\end{abstract}

Key words: Particle generation, Powder packing, Growing particle, Discrete Element Method 


\section{Introduction}

Powder is widely used and traditionally applied to various fields such as in agriculture, chemical industries, and civil engineering. Recently, it has been applied to pharmaceutical, cosmetics, 3D-printing, particulate composites, and battery industries where performance efficiency varies depending on particle packing. In the pharmaceutical field, studies on random particle packing for modeling pharmaceutical manufacturing processes using X-rays and scanning electron microscopy have been conducted [1], and in the field of 3D printing, research is being conducted for additive manufacturing of materials [2]. Thus, many studies have been conducted to improve the reliability of packing results in these fields. In these studies, the results of random packing of dodecahedron particles of various sizes with theoretical results [3] or numerical results were compared with the effect of particle shapes on the wettability of electrodes using the lattice Boltzmann method (LBM) to improve the efficiency of the battery [4]. Powder packing research is being actively conducted to increase efficiency by developing methods to improve compression in the limited space of the cathode and anode materials of a battery.

There has also been progress in fundamental research to expand the application range of powder technology. Studies have reported on the porosity of powder mixed with spherical particles of various sizes [5], classified the contact patterns of free fall particles using coordination number $(\mathrm{CN})$ to determine the degree of packing between particles [6], and investigated the effect of particle size on packing by analyzing results using packing density [7]. Furthermore, studies attempted to arbitrarily conduct packing of non-spherical powders by modifying the algorithm used to combine elliptical particles with spherical particles [8], compare granulator experiment and simulation results to determine the effect of water on the forces acting between particles [9], and described the effect of a fluid on the fluid/powder mixture using as a new force rather than the existing capillary force [10].

The most widely used particle-based simulation method in this powder packing study is the discrete element method (DEM), which was first proposed by Cundall and Strack in 1979 [11]. Since then, research to determine various parameters through experiments has been actively conducted to increase the accuracy of DEM [12]. He et al. [13] used DEM to analyze the effects of compressive strength and failure pattern by applying contact force, capillary force, and solid bridge model to particle generation, stack, and powder packing; Curtis et al. [14] conducted a study comparing the experimental results with random packing simulation results after connecting several particles together to form a cylindrical rod. Xiao et al. [15] used a contact force model in which attractive forces between particles, such as van der Waals forces that act on fine particles, were included to find powder packing densities that could vary depending on particle size, aspect ratio of the particle, and surface energy acting on the particle surface. Partelil et al. [16] demonstrated through experiments and DEM analysis that accurate analyses can be performed only when adhesion and non-bonded van der Waals forces are considered in the packing of 4-52 $\mu \mathrm{m}$ powders. In addition, Lee et al. [17] used a mass scaling method to solve the very-small timestep problem of small particles used in additive manufacturing.

In general, the most challenging problem with DEM is the long computation time. The Rayleigh timestep, which calculates the propagation rate of stress waves in solids, is used to obtain an optimum time step in the DEM [18]. However, the Rayleigh time step is proportional to the size of the particle and inversely proportional to the elastic modulus. Therefore, the analysis time increases exponentially when the particle size decreases or the elastic modulus increases. Therefore, in a study conducted by Joanna et al. [19], a large Rayleigh time step was applied using an exceptionally large particle size of $7.3 \mathrm{~mm}$ and an unrealistic elastic modulus of 560MPa to satisfy the time constraints [19]. Thus, if the particle size for analysis is in the submicron range, the time step is dramatically reduced and the time to analyze the behavior of the powder is excessively increased. Accordingly, Haervig et al. [20] proposed a model in which the properties of particles are modified to reduce the time constraints of the simulation owing to the size of the time step. However, because only a slight difference in results between 
the model using the modified expression and the existing model was found, the problem is yet to be completely solved.

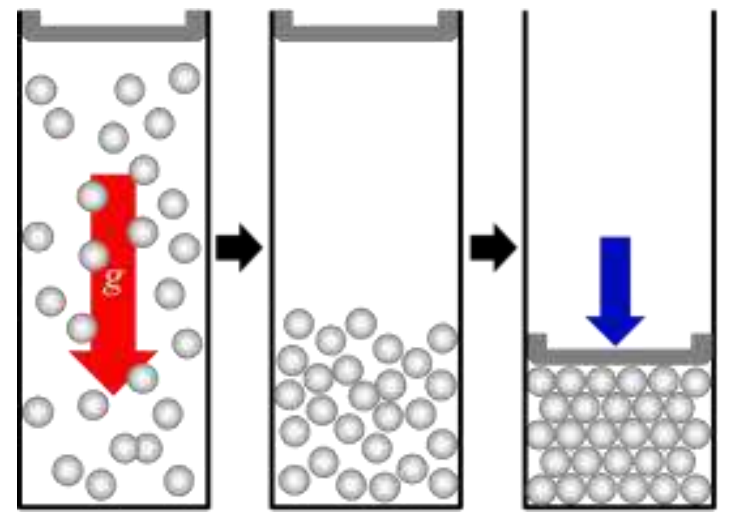

(a) Free fall method

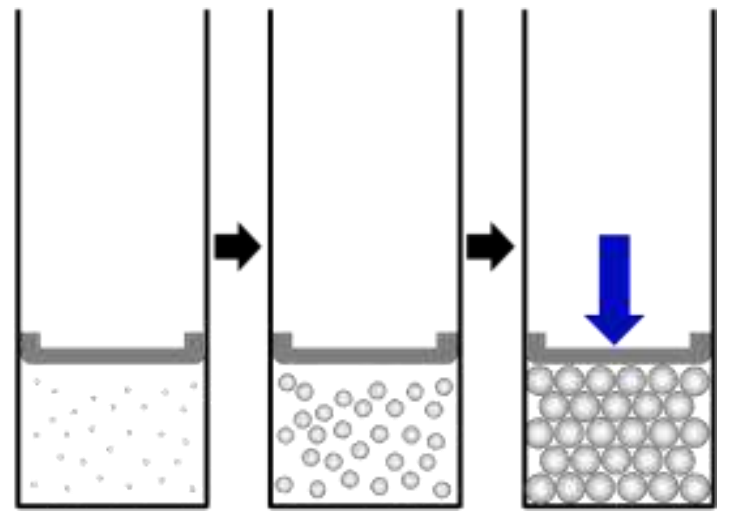

(b) Growing particle method

Fig. 1 Schematic of free fall model and growing particle model

Various efforts have been invested to solve problems caused by the reduction of computation time in the DEM. Recently, studies on the optimization of updated timesteps in the nearest neighbor method have been conducted [21], and the coarse graining method, which combines several particles into one particle in the calculation, has been proposed [22, 23].

However, although the coarse graining method reduces computation time, it does not solve the time loss that occurs during the early stage of the simulation when particles are generated. In the general DEM analysis, as shown in the free fall model in Fig. 1(a), the analysis progresses as follows: particle generation; stacking by gravity; and packing. Parteli et al. [16] conducted a study that compares the simulations and packing experiments for very-small glass particles. To conduct powder packing in the simulation process, particles were first generated in a wider space and then powder packing was performed using gravity. Yang et al. [24] conducted simulations in the order as shown in Fig. 1(a) to confirm the packing structure of uniform powder, which was conducted when van der Waals strength was dominant. In most cases, while the particle generation/gravity stacking process is an unnecessary process for the analysis, their computation time is very long. Dong et al. [25] generated and stacked powder over about $2 \mathrm{~s}$ to form a cake when the precipitation and filtration processes proceed at a uniform flow rate or pressure [25]. However, the actual simulation process required for the study occurs afterward, and the computation time for obtaining results is less than $2 \mathrm{~s}$. Therefore, current methods waste simulation time in unnecessary steps. To solve this problem, Cassares et al. [26] proposed a method to minimize computation time by stacking layers using a layer-by-layer method, and Donze et al. [27] attempted to minimize computation time by generating particles in the tetrahedral mesh in the finite element method.

Therefore, a new method that can shorten the simulation time while maintaining the physical properties and size of the particles is proposed in this paper. The method is targeted at powder packing simulations that are frequently studied in fields such as batteries and civil engineering. The traditional packing simulation method is called a free fall packing model, and packing is performed through "particle generation," "stack and stabilization by gravity," and "compression." The growing particle model method shown in Fig.1(b) was employed to attempt minimizing the analysis time. The growing particle model method generates fine particles in a limited space, and then increases the size of the particles to fill the space. The results of this packing simulation method were compared with those of the existing packing simulation method. Further, the proposed method was applied to various contact models used in the DEM, and the applicability of the proposed method for each contact model was examined.

\section{Results}


The Contact Number $(\mathrm{CN})$, porosity, and RDF are generally used to compare the internal structure of particle contact and packing models. Therefore, this paper also examined the averaged $\mathrm{CN}$, average porosity, and RDF to examine the packing structure in the container and confirmed the spatial distributions of the local $\mathrm{CN}$ and porosity in the container.

\subsection{Averaged Coordination Number $(\mathrm{CN})$ and Porosity}

The $\mathrm{CN}$ is the number of particles within a certain radius around a reference particle and this number indicates the structure of powder packing. The radius can be defined as the ratio of the diameter of the reference particle, and when the radius is $1.01 d_{p}$, this represents the number of particles in contact with the reference particle. If all particles are the same size and particle deformation can be ignored, the theoretical maximum $\mathrm{CN}$ is 12 with a radius of $1.0 d_{p}$. However, since particle deformation should be ignored when the radius is $1.0 d_{p}$, many studies use a radius of $1.005 d_{p}, 1.01 d_{p}$, or $1.0 d_{p}[28,29]$. Therefore, in this paper, the powder packing structure was confirmed by using a radius of $1.01 d_{p}$ used frequently.

The measured CNs are almost constant regardless of the contact model or packing model and the values are about 6.3 with only 0.033 standard deviation. This is similar to the FF model where particles stack up and the GP1 and GP2 models where particles are filled in a confined space, which indicates that there is no significant difference in the internal structure due to contact between particles when packing is performed.

Porosity is the ratio of the empty volume within a space. In other words, this is the volume ratio of the container to the pores excluding the volume of the particles of the powder in the container, and this can be calculated as

$$
\phi=\frac{V_{V}}{V_{T}}
$$

where $V_{V}$ is the volume of the pore and $V_{T}$ is the entire volume. Furthermore, in this paper, the porosity was calculated by excluding the overlapped volume and considering the contacted and overlapped particles for the accurate calculation of the pores. However, because the entire volume of the container is constant in all cases, the sum of the calculated overlaps for all particles is very small, the same powder compression rate is used in all cases, and the macroscopic porosity shows almost the identical values, 0.387 .

\subsection{Radial Distribution Function (RDF)}

The RDF is one of the important parameters to understand the internal structure as it analyzes the packing structure that shows how many particles are concentrated around the reference particle as a function of distance [30]. An RDF value, the number density of particles that exist between two virtual spherical spaces with a radius $r$ and $r+d r$, is defined as

$$
g(r)=\frac{d N(r)}{4 \pi r^{2} \rho d r}
$$

where $d N(r)$ indicates the physical number of particles that exist between two virtual spherical spaces having radii $r$ and $r+d r . \rho$ is the number density of the particles and can be calculated as $\rho=N / V$, where $N$ is the number of particles in the virtual sphere with radius $r$, and $V$ is the volume of the virtual sphere. In Equation (2), $4 \pi r^{2} d r$ is the volume between the virtual spherical space with a radius $r+d r$ and the virtual spherical space with a radius $r$. Therefore, Equation (2) becomes

$$
\mathrm{g}(\mathrm{r})=\frac{N_{r+d r}-N_{r}}{V_{r+d r}-V_{r}} \times \frac{V_{r}}{N_{r}}
$$

In general, if the particles are the same and completely spherical, the RDF peak values appear at 1.0, 1.73, and 2.0 radial distances, which represent multiples of the particle diameter. If the particles are adjacent to each other, this value becomes 1.0, and if three particles form a triangular structure, the value becomes 1.73 [30]. Finally, if three particles are in a straight line, the value becomes 2.0 . 


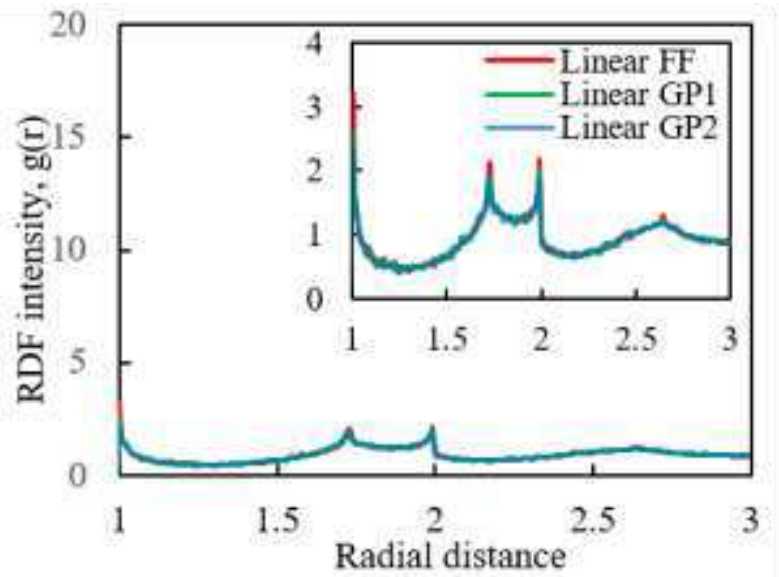

(a)

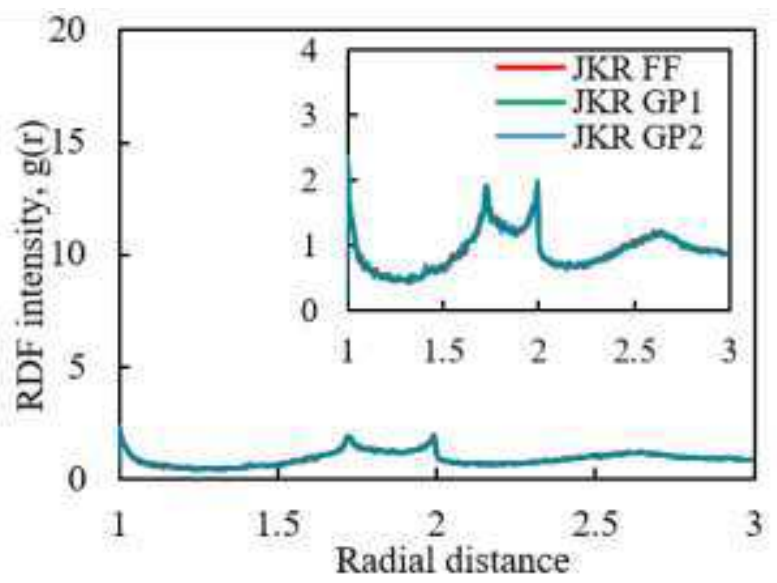

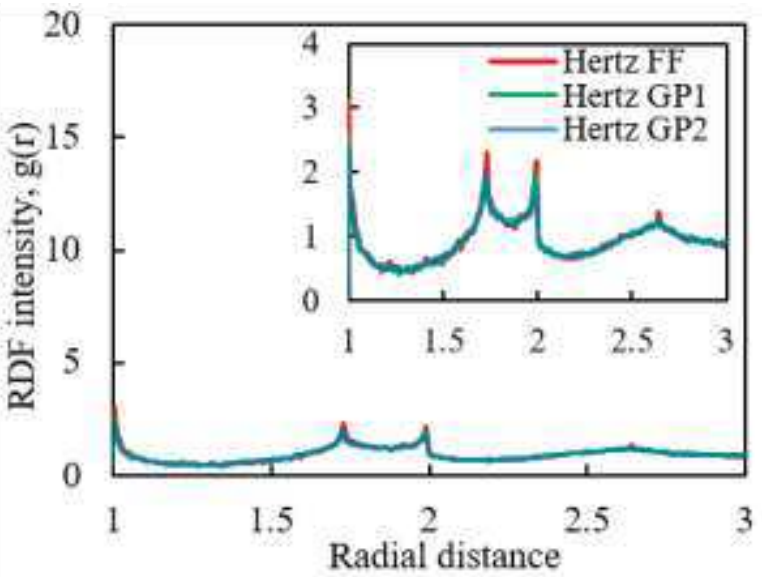

(b)

(c)

Fig. 2 Dependence of RDF between different contact model and packing model; (a) Linearspring contact model with various packing model, (b) Hertz-Mindlin contact model with various packing model, (c) Hertz-Mindlin with JKR model with various packing model.

As shown in Fig. 2, the peak values in this study are observed at nearly the same three radial distances regardless of the contact model. Furthermore, in each contact model, all packing models show almost the same intensity. This indicates that all three packing models (FF, GP1, and GP2) show almost identical packing structures between particles regardless of the contact model. In other words, in terms of the RDF, there is almost no difference between the packing models.

Fig. 2 (a) shows the RDF graph when the linear-spring contact model is used and the packing model is applied differently. The radial distances in all packing models show peak values at 1.0, 1.73, and 2.0. Although the intensity of the free fall packing model is slightly higher than that of other packing models, the difference is negligible. Fig. 2 (b) and (c) show the results when three different packing models are applied to the Hertz-Mindlin contact model and Hertz-Mindlin following JKR contact model. As shown in Fig. 2(a), the two graphs have peak values at the same radial distances and have almost the same intensity. Finally, Fig. 2 shows that in one contact model, particles have the same packing structure regardless of the packing model. 


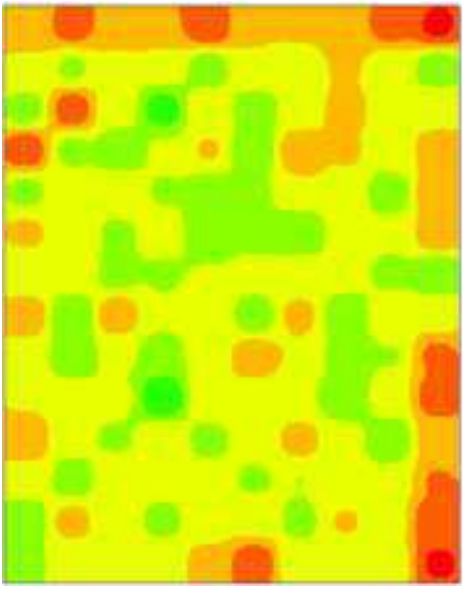

(a) Linear-FF model;

Linear-GP2 model;

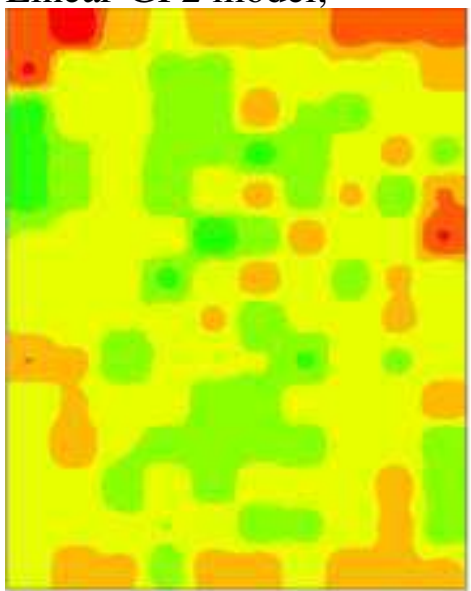

(d) Hertz-FF model;

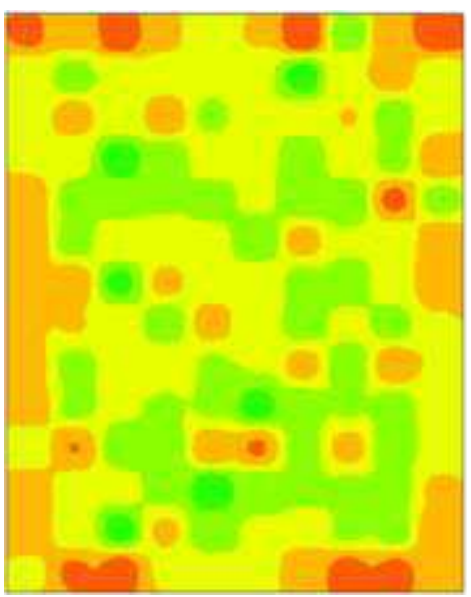

(g) JKR-FF model;
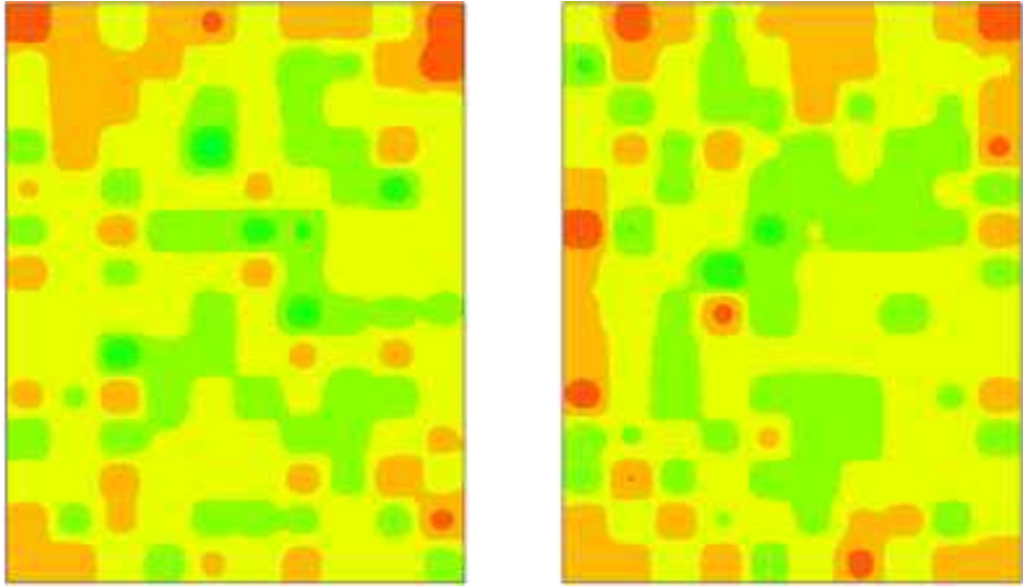

(b) Linear-GP1 model;

(c)

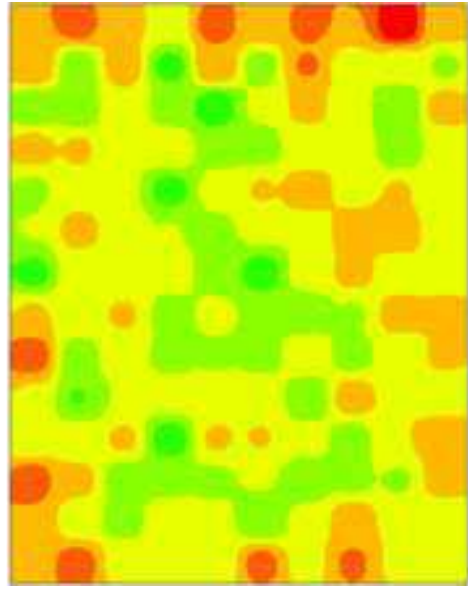

(e) Hertz-GP1 model;

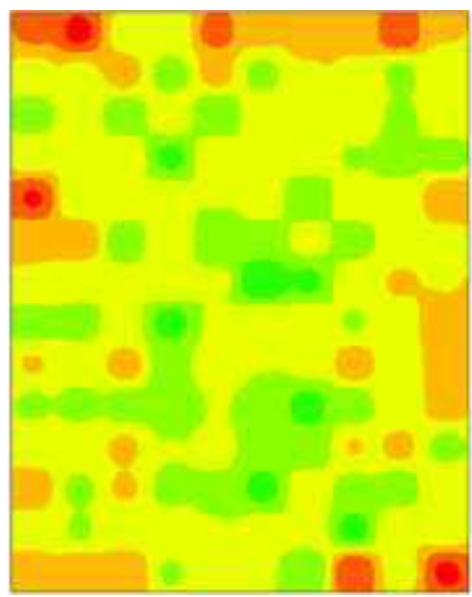

(h) JKR-GP1 model;

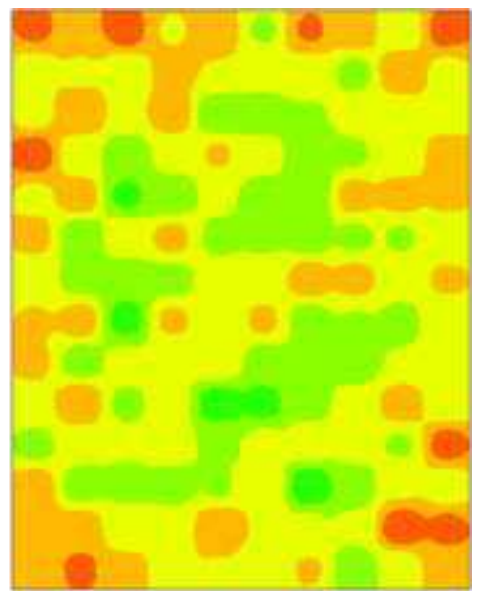

(f) Hertz-GP2 model;

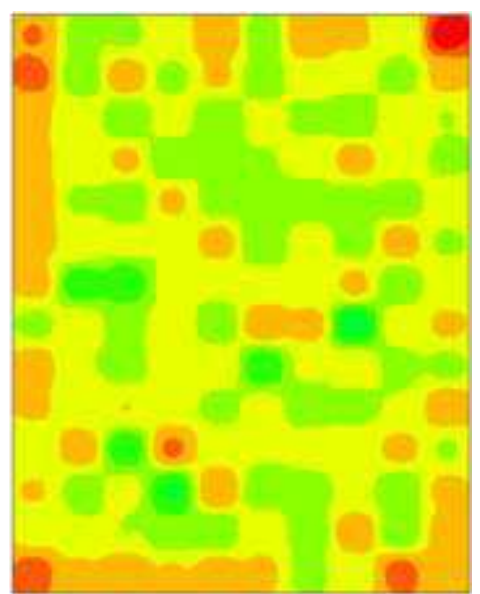

(i) JKR-GP2 model;

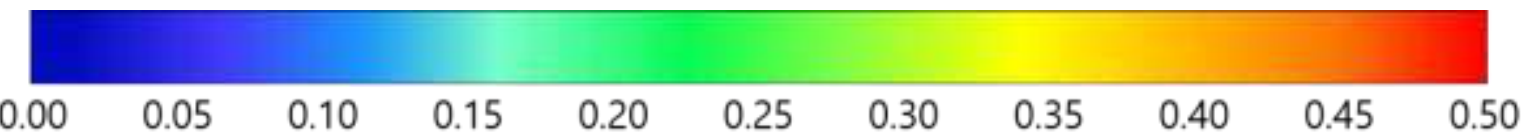

Fig. 3 Distribution of porosity for different contact model and packing model 


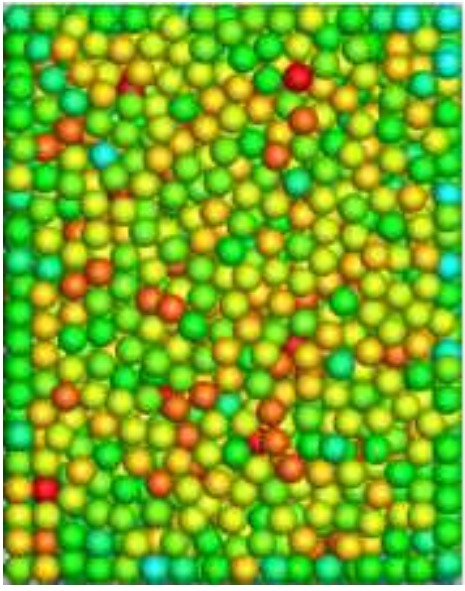

(a) Linear-FF model; model;

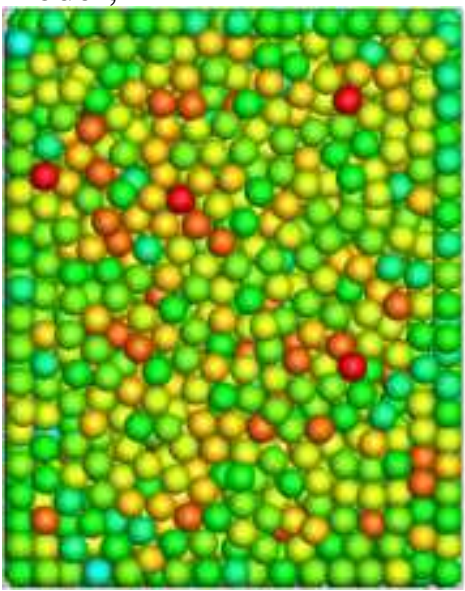

(d) Hertz-FF model;

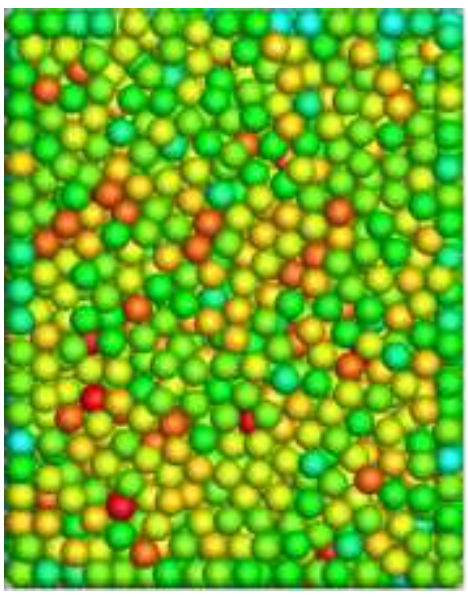

(g) JKR-FF model;

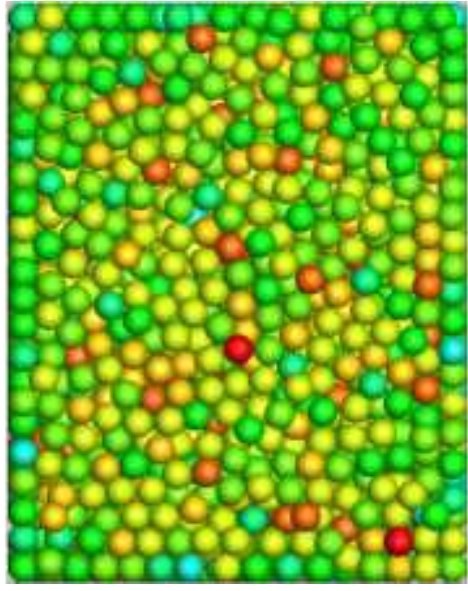

(b) Linear-GP1 model;

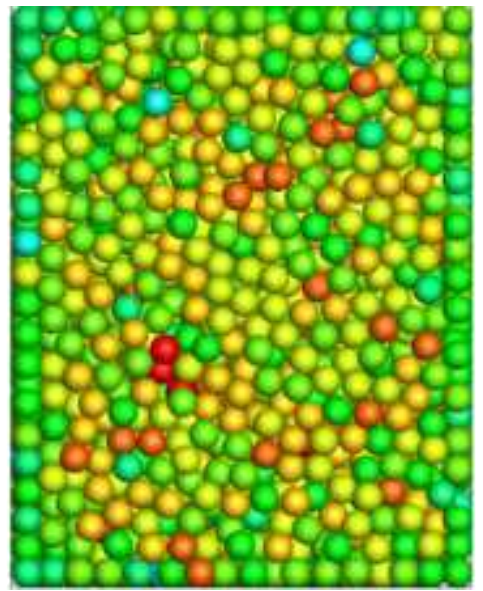

(e) Hertz-GP1 model;

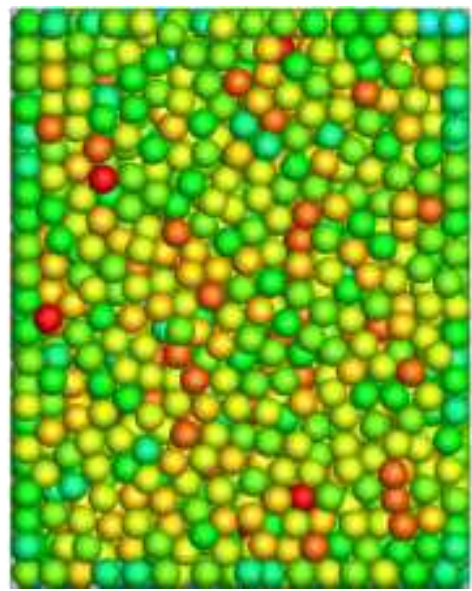

(h) JKR-GP1 model;

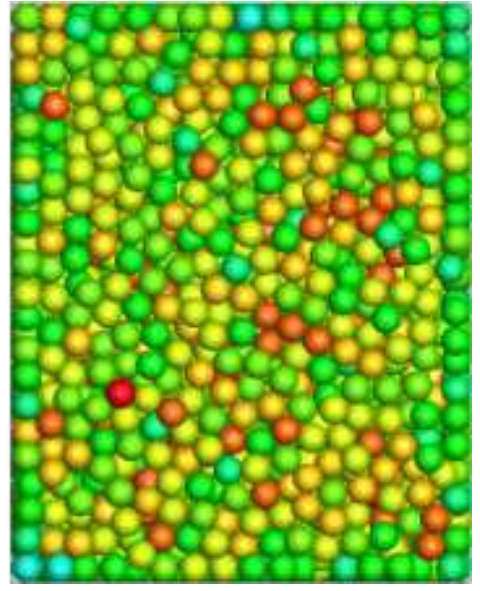

(c) Linear-GP2

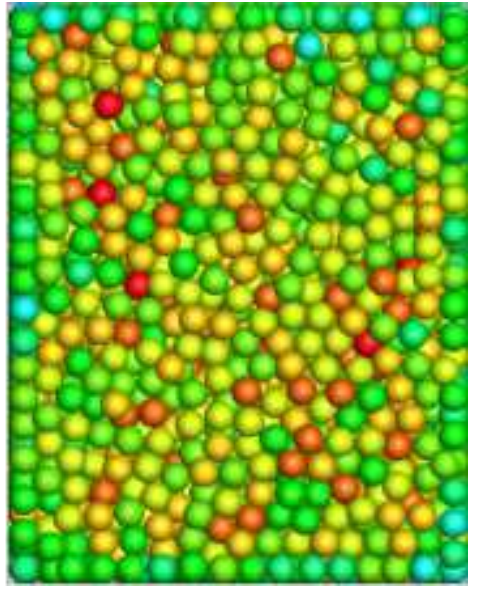

(f) Hertz-GP2 model;

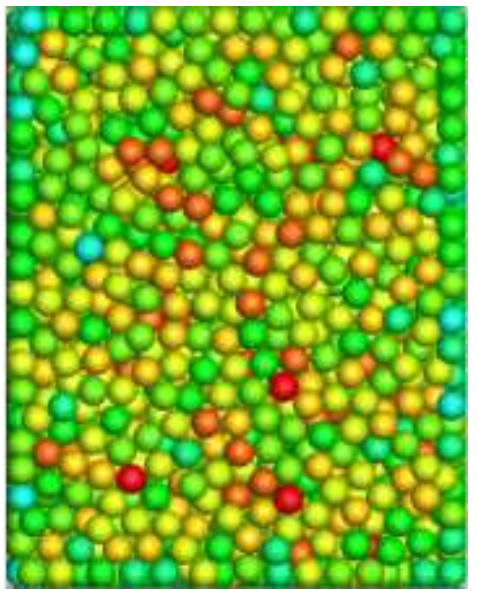

(i) JKR-GP2 model

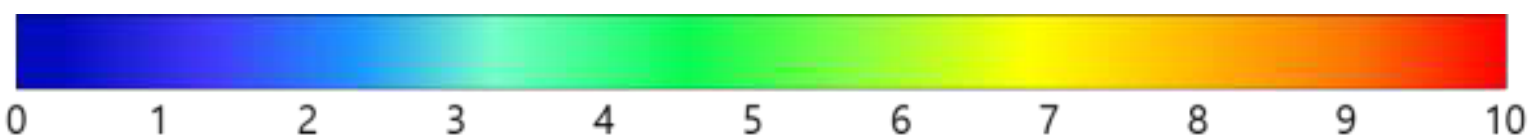

Fig. 4 Dependence of contact number between different contact model and packing model 


\subsection{Spatial distribution of porosity}

Although average porosity can determine the degree of overall packing, it is difficult to understand the spatial distribution of pores. Therefore, the compressed container is divided into several regions and the porosity of each region is examined. In this paper, the length and width are divided into 10 sections each, and the height is divided into 14 sections to form cuboid regions; then the porosity of each region is calculated. Fig. 3 shows the porosity distributions when all contact and packing models were used. Because it is a nearly symmetrical distribution, only the $\mathrm{x}-\mathrm{y}$ section around the center point in the z-axis direction is shown. Although the pore distribution can be slightly different depending on the contact model or the packing model, in general, the porosity is large at the edge of the wall, the particles are well packed, and the porosity is low around the center. In all cases the smallest porosity is about 0.25 and the largest porosity is about 0.5 . Errors were checked for each contact model to determine differences in porosity depending on the packing model. The average porosity of the structure using the linear spring contact model and the free fall packing model is 0.377 . Based on this value, the average porosity error between the Linear-GP1 and Linear-GP2 models is $0.57 \%$ and $0.94 \%$, respectively. The error of the packing simulation in three ways using the Hertz-Mindlin contact model was also based on the FF model. The Hertz-FF model had a porosity of 0.380 , HertzGP1 and Hertz-GP2 were shown an error of $0.61 \%$ and $0.93 \%$, respectively. Finally, in the case of the Hertz-Mindlin following JKR contact model, the JKR-GP1 model showed an error of $0.38 \%$ and the JKR-GP2 model showed an error of $0.7 \%$ based on the JKR-FF model. The porosity of the JKR-FF model was 0.376 . Overall, the porosity of the simulation result using the Growing particle model has an error within $1 \%$ from the porosity of the simulation result using the free fall model.

In addition, the JKR model (Fig. 3 (g), (h), and (i)), which considers the attractive force between particles due to the influence of surface energy, and a model that does not consider the attractive force (Fig. 3 (a), (b), (c), (d), (e), and (f)) show no significant differences in the pore distribution. This is because the powder compression ratio is so large that the effect of the attraction force between particles is almost negligible.

\subsection{Spatial distribution of contact number}

Like porosity, the spatial distribution of the $\mathrm{CN}$, which can represent the internal structure of the packing, was also confirmed. Fig. 4 is a projection of the center point in the Z-axis direction on the $\mathrm{x}-\mathrm{y}$ plane, similar to Fig. 3. Like porosity, particles with high CNs are distributed around the center of the packing structure, and particles at the edges generally have low CNs. Similar to the comparison results for porosity, the average and error of the $\mathrm{CN}$ values were confirmed. The average $\mathrm{CN}$ of the Linear-FF model is 6.28 , and based on this value, the error of LinearGP1 is $0.32 \%$ and the error of Linear-GP2 is $0.40 \%$. The average CN for the Hertz-FF model is 6.23 , and based on these values, the errors for Hertz-GP1 and Hertz-GP2 are $1.33 \%$ and $1.39 \%$, respectively. Finally, the CN of the JKR-FF model is 6.26 , the error of JKR-GP1 is $0.47 \%$, and the error of JKR-GP2 is $0.18 \%$. Although the error in the Hertz-Mindlin contact model is slightly larger than that in other simulations, it shows a value of $1 \%$, and therefore, it can be said that the two growing particle packing methods are valid. 


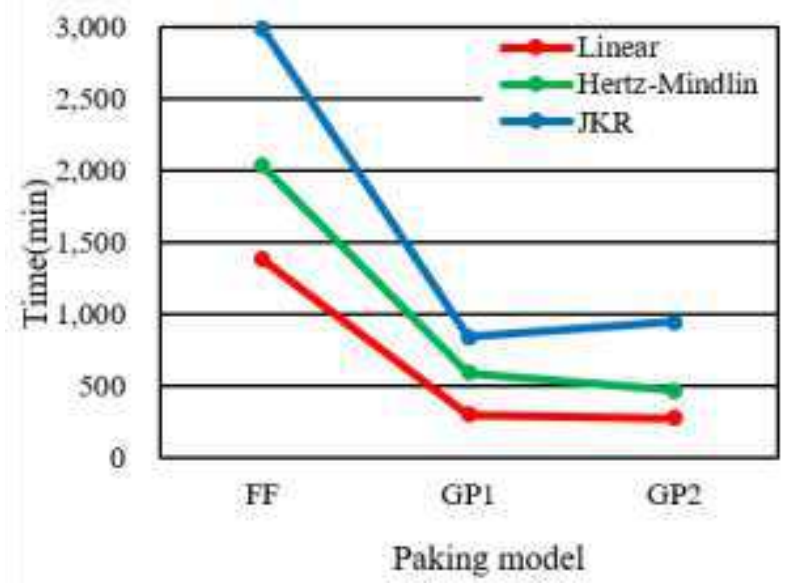

Fig. 5 Comparison to simulation running time between packing models

\subsection{Simulation total time}

Fig. 5 shows the calculation times for three contact models and three packing models used in this paper. Even in the simple packing simulation, the simulation time can vary greatly depending on the number and properties of the particles. Furthermore, the simulation calculation time can vary depending on the type of contact model used in the simulation. Fig. 5 shows that the computation time of the linear spring contact model is shorter than that of the Hertz-Mindlin model or the JKR model. It was also found that the computation time of the JKR model, which calculates the surface attraction, is longer than that of the Hertz-Mindlin model. According to the packing model results, for the free-fall model analysis using a single CPU (i7-6700K), the computation time of the JKR contact model was the longest (about 3,000 min), while the computation time of the linear spring contact model was the shortest (about 1,400 min). However, in the model where particles are filled and grown in a limited space such as the GP1 and GP2 packing models, the calculation time was reduced to $400 \%$ of the free fall packing model on average. In other words, the computation time of the FF model is almost four times longer than that of the GP1 model or GP2 model.

\section{Discussion}

This study was conducted to minimize the analysis time of the simulation of DEM-based powder packing. Therefore, the FF, GP1, and GP2 models were compared. Furthermore, to examine the effect of the contact model, the linear, Hertz-Mindlin, and JKR models, were used. The average porosity, average $\mathrm{CN}, \mathrm{RDF}$, spatial porosity, and spatial $\mathrm{CN}$ were analyzed to find the difference in the packing structures according to the packing model. Since all packing models showed almost identical porosity, CN, RDF, and spatial distribution, the differences according to the packing models are negligible. This indicates that the growing particle model can replace the existing free fall model. Furthermore, among the two growing particle models, the GP1 model was closer to the free fall model than the other model due to the slight difference in the spatial CN distribution; however, the difference is almost negligible.

Therefore, in this study, it was proposed to use the growing particle model instead of the free fall model because it can greatly reduce the computation time. In particular, the growing particle model would become more useful as the total number of particles increases or the time step for analyzing the behavior of particles decreases by using more rigid materials.

Although the growing particle model was applied only into the particle packing simulation, it can be expanded to other simulation based on DEM. Especially, in complex domain, it can be quite advantageous if certain parts need to be emptied and the rest filled. These techniques have not been applied to commercial software such as EDEM and Rocky. But, we believe that this technology will greatly help reduce the simulation time in the future if applied. 


\section{Methods}

\subsection{Contact Force Model for DEM}

In this paper, a linear spring model and a Hertz-Mindlin model - the most widely used nonlinear spring models - are used to understand the effect of the contact force model that calculates contacts between particles on the simulation; a Hertz-Mindlin model employing the JKR theory that includes the Johnson-Kendall-Roberts model (JKR model) and the effect of the surface force in the analysis is also used.

The linear spring model [11] calculates the contact force between particles using the spring stiffness and damping coefficients of the particles. The force has two components: a normal force, which is a force acting perpendicular to the particle motion, and the tangential force, which is a force acting along the particle motion. These forces can be calculated as

$$
\begin{aligned}
& F_{n}=-k_{n} \delta_{n}+v_{n} \Delta v_{\text {contact }} \\
& F_{t}=\min \left(k_{s} \delta_{t}+v_{s} \Delta v_{\text {contact }}, \quad \mu_{s}\left|F_{n}\right|\right)
\end{aligned}
$$

where $F_{n}$ is the normal force acting perpendicular to the particle, and $k_{n}, v_{n}, \delta_{n}, \Delta v_{\text {contact }}$, and $\mu_{s}$ are spring stiffness coefficient in the normal direction, damping coefficient, overlapping distance between particles (i.e., overlap), relative velocity between particles in the normal direction, and coefficient of friction, respectively. Further, $k_{n}$ can be calculated by using Hertzian strain energy as

$$
k_{n}=\frac{16}{15} r^{* \frac{1}{2}} E^{*}\left(\frac{15 m^{*} v^{2}}{16 r^{*} \frac{1}{2} E^{*}}\right)^{\frac{1}{5}}
$$

where $r^{*}, E^{*}$, and $m^{*}$ are equivalent radius, equivalent Young's modulus, and equivalent mass, respectively.

The damping coefficient of the particle $v_{n}$, can be calculated as

$$
v_{n}=\sqrt{\frac{4 m^{*} k_{n}}{1+\left(\frac{\pi}{\ln e_{p}}\right)^{2}}}
$$

where $e_{p}$ is the repulsive coefficient of the particle.

The Hertz-Mindlin model is one of the most widely used models in the DEM [31]. The normal force can be calculated as

$$
F_{n}=\frac{4}{3} E^{*} \sqrt{r^{*}} \delta_{n}^{\frac{3}{2}}-2 \sqrt{\frac{5}{6}} \beta \sqrt{S_{n} m^{*}} \Delta v_{\text {contact }}
$$

where $\beta=\ln e_{p} / \sqrt{\ln ^{2} e_{p}+\pi^{2}}$ and $\mathrm{S}_{\mathrm{n}}=2 E^{*} \sqrt{r^{*} \delta_{n}}$. The tangential force is defined as

$$
F_{t}=-S_{t} \delta_{t}-2 \sqrt{\frac{5}{6}} \beta \sqrt{S_{t} m^{*}} \cdot \Delta v_{\text {contact }} \cdot \hat{s}
$$

where the tangential stiffness factor $S_{t}$ is defined as $S_{t}=8 G^{*} \sqrt{r^{*} \delta_{n}}, G^{*}$ is the equivalent shear modulus, and $\delta_{t}$ is the overlap in the tangential direction. Furthermore, similar to the linear spring model, the $F_{t}$ value is compared to the Coulomb friction value and the smaller value is used.

The JKR theory is a contact theory that calculates adhesive force using the loss balance between the stored elastic energy and surface energy to include the effect of the attraction force (i.e., adhesion force in the Hertz contact theory) in the analysis [32]. The Hertz-Mindlin model following the JKR theory can be expressed as

$$
\mathrm{F}_{\mathrm{n}}=-\frac{4}{3} E^{*} \sqrt{r^{*}} \delta_{n}^{\frac{3}{2}}-2 \sqrt{\frac{5}{6}} \beta \sqrt{S_{n} m^{*}} \Delta v_{\text {contact }}+\sqrt{16 \gamma E^{*} \pi a^{3}}
$$

where $\gamma$ is the surface energy of the particle and $a$ is the contact radius of the particle. In the above equation, the first and second terms are same as that in the general Hertz-Mindlin model, and the last term is the JKR term that represents the effect of the attraction force. When the JKR term is added, the surface area becomes larger than the surface area predicted by Hertz's 
theory, and even when the load is removed, the contact area exists and this will indicate the effect of attraction.

Table 1 Material properties

\begin{tabular}{lc}
\hline Particle parameter & Value \\
\hline Particle size, $r_{p}$ & $25 \mu \mathrm{m}$ \\
\hline Particle density, $\rho$ & $2500 \mathrm{~kg} / \mathrm{m}^{3}$ \\
\hline Total number of particles & 5000 \\
\hline Young's modulus, $E$ & $6.3 \times 10^{9} \mathrm{~Pa}$ \\
\hline Poisson's ratio, $v$ & 0.24 \\
\hline Coefficient of restitution, $\varepsilon_{p}$ & 0.95 \\
\hline Friction coefficient (particle to particle), $\mu_{p}$ & 0.1 \\
\hline Friction coefficient (particle to wall), $\mu_{w}$ & 0.0 \\
\hline Surface energy (only for JKR model), $\gamma$ & $0.0199 \mathrm{~N} / \mathrm{m}^{2}$ \\
\hline Wall parameter & \\
\hline Upper plate velocity & $-2 \mathrm{~mm} / \mathrm{s}$ \\
\hline Compaction ratio & 0.943 \\
\hline
\end{tabular}

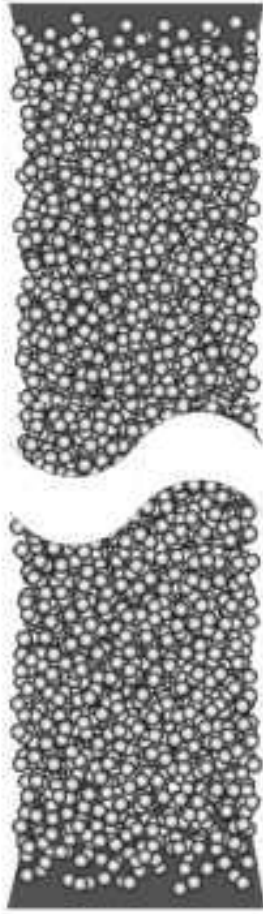

(a) $0.0 \mathrm{~s}$

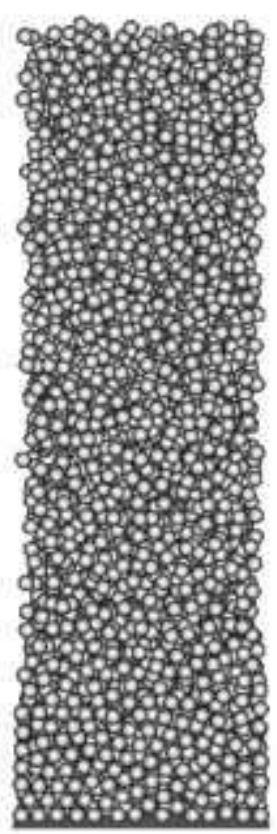

(b) $0.01 \mathrm{~s}$

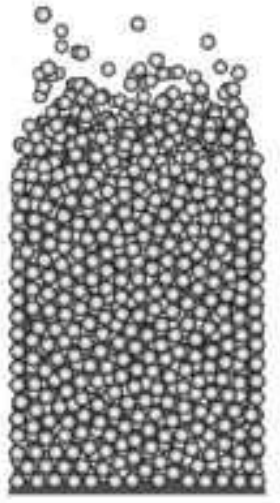

(c) $0.02 \mathrm{~s}$

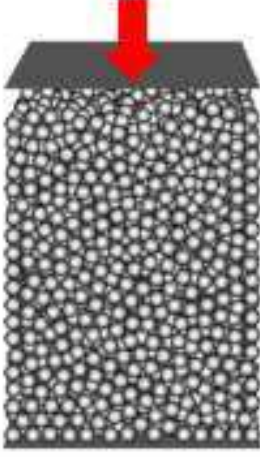

(d) $0.05 \mathrm{~s}$

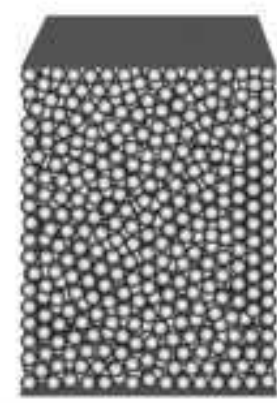

(e) $0.15 \mathrm{~s}$

Fig. 6 Snapshots for free fall particle packing: (a) $0.0 \mathrm{~s}$; (b) $0.01 \mathrm{~s}$; (c) $0.02 \mathrm{~s}$; (d) $0.05 \mathrm{~s}$; (e) $0.15 \mathrm{~s}$.

\subsection{Simulation setup}

The structure of particles is usually analyzed by filling a large number of particles in a container and compressing them in the powder packing simulation using DEM; this same analysis method is used in this study. Three contact force models (linear spring, Hertz-Mindlin spring, and Hertz-Mindlin spring following JKR theory), and three packing methods (free fall model, growing particle model with full gravity: GP1, and growing particle model with partial gravity: GP2) are used. Therefore, a total of nine models $(3 \times 3)$ are used in this study. 
The container used in this study is a cuboid measuring $0.375 \mathrm{~mm} \times 0.375 \mathrm{~mm} \times 1.675 \mathrm{~mm}$ $(\mathrm{L} \times \mathrm{W} \times \mathrm{H})$. The particle size was set to $25 \mu \mathrm{m}$ so that the ratio of the length and width of the container can be set at 15: 1, and the height of the container was set high enough to avoid initial overlap problems during random generation and fall of particles. The particle distribution was monodispersed to simplify the simulation. Other properties of the container and the particle are summarized in Table 1.

As shown in Fig. 6, in the free-fall model using the Hertz-Mindlin contact model, a total of 5000 particles are randomly generated in the container once the simulation starts (Fig. 6 (a)). In this simulation, the initial porosity was set excessively high to 0.826 , and the initial velocity was set to 0 to prevent the particles from overlapping each other. Particles fall and are stacked under the influence of gravity (Fig. 6 (b)). In this process, the particles collide with other particles and walls and lose energy, and the particle velocity is eventually stabilized (Fig. 6 (c)). At the end of the stabilization process, the plate on the top of the container descends at a constant speed $(2 \mathrm{~mm} / \mathrm{s})$ to compress the powder (Fig. 6 (d)). After the stabilization process, the powder was only compressed up to about $6 \%$ of the height of the accumulated powder. The plate that compresses the particles remains stationary after the compression process (Fig. 6 (e)). This packing process can be divided into the order of particle generation-stack and stabilization-compression, and in this paper, it is called a free fall model.

Although the physical time in the simulation using the DEM is different for each contact model and packing model, packing in the given condition is completed in about $0.13 \mathrm{~s}$. Therefore, all simulation results were measured after $0.15 \mathrm{~s}$. The time step in the simulation was determined by the critical Rayleigh time step presented by Kremmer et al. [18] as

$$
\Delta \mathrm{t}_{\mathrm{crit}}=\frac{\pi r_{\min }}{\Phi} \sqrt{\frac{\rho}{G}}
$$

where $r_{\text {min }}$ is the radius of the smallest particle, $\rho$ is the particle density, $G$ is the shear modulus of the particle, $\Phi=0.163 v+0.8766$, and $v$ is the Poisson's ratio. In this study, the time step was set to $1.0 \times 10^{-8} \mathrm{~s}$, which is about $30 \%$ of the critical time step, to reduce the computational error that can occur during the simulation.

In the existing method, where the compression process occurs after the free fall, most simulation time is spent on meaningless processes including free fall and compression processes. Therefore, in this paper, the growing particle model, which eliminates the free fall and compression processes of the particle and gradually grows the particle in a defined space, is proposed.

\subsection{Free Fall Packing Model (FF)}

The free-fall model is one of the most widely used models in existing research on packing $[16,24,25]$. As described above, after particles are generated in the limited space of a container, the freely falling particles are stacked by gravity. Then, a plate is used to add load on the particles at a constant speed to compress them to the desired depth. The shape at each stacking step of the free-fall model is already shown in Fig. 6, even according to the three different contact force models (Linear model: Linear, Hertz-Mindlin model: Hertz, Hertz-Mindlin and the following JKR theory: JKR), the difference in shape is almost negligible after the load is applied. Comparison with the shape before this application is meaningless. Therefore, all results are compared to the shape after the load is applied.

\subsection{Packing Model for Growing Particle with Full Gravity (GP1)}

To reduce the overall computation time, very small particles were generated in the initial step and the space was gradually filled by increasing the particle size in a very short time. Small particles were generated in the first step to avoid initial overlaps that can cause abnormally large contact forces. In other words, instead of dropping and compressing the generated particles into the limited space, small particles were initially generated and expanded to fill the 
limited space. Therefore, the height of the upper plate was set equal to the final height that will be reached by the plate in the free fall packing model.

The initial size of the particle can be expressed in terms of the ratio of the actual radius of the particle to be analyzed, and it was set to $r_{0}=\xi_{r} r_{p, t a r g e t}$, where $\xi_{r}$ is the ratio of the size of the particles generated at the beginning of the simulation, and it was set to 0.01 . In other words, the size of the generated particles is $1 / 100$ of the desired size. After that, the size of the particle is increased using the exponential function shown below.

$$
\begin{array}{ll}
r_{p}=r_{p, \text { target }}\left(1+\xi_{r}-e^{\left(-\frac{t}{\xi_{r} \tau}\right)}\right), & \text { if } r_{p}<r_{p, \text { target }} \\
r_{p}=r_{p, \text { target }}, & \text { if } r_{p} \geq r_{p, \text { target }}
\end{array}
$$

where $r_{p}$ is the radius at the current time step of the given particle, $r_{p, t a r g e t}$ is the radius of the particle when the particle size is the largest (i.e., the desired radius of the particle), $t$ is the current time, and $\tau$ is the slope of the exponential function (i.e., the rate at which the size of the particles grow). In this study, $\tau$ is set to 0.1 ; by adjusting this value, the rate of the particle size can be controlled.

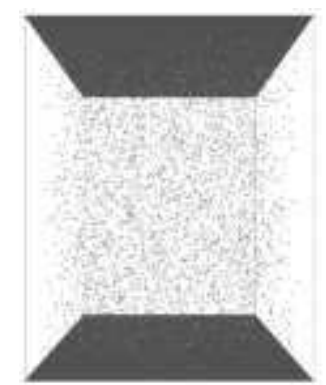

GP1: (a) $0.0 \mathrm{~s}$

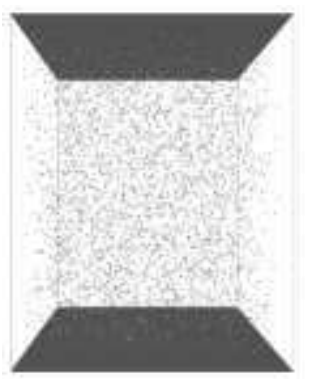

GP2: (f) $0.0 \mathrm{~s}$

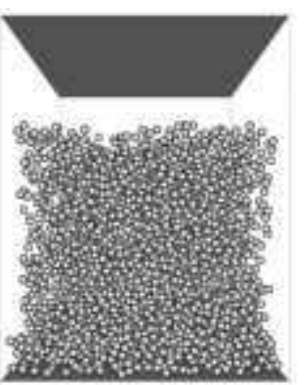

(b) $0.005 \mathrm{~s}$

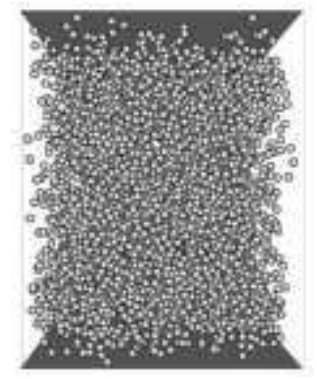

(g) $0.005 \mathrm{~s}$

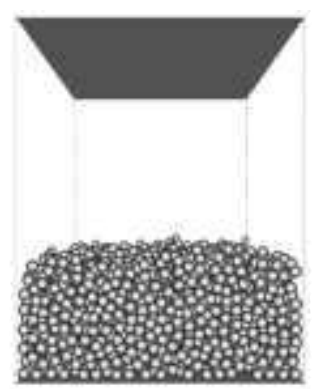

(c) $0.0082 \mathrm{~s}$

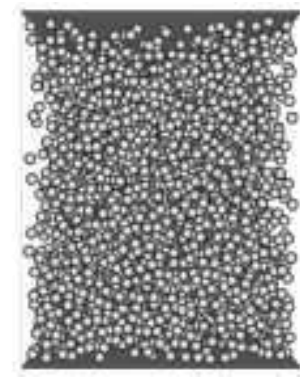

(h) $0.0082 \mathrm{~s}$

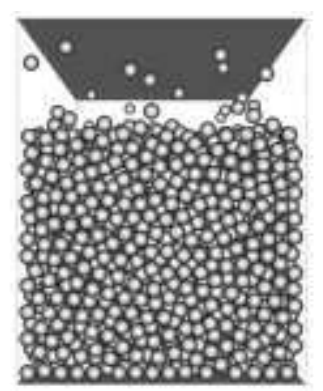

(d) $0.015 \mathrm{~s}$

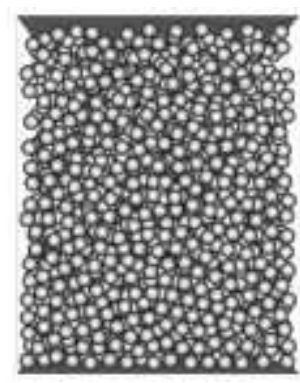

(i) $0.015 \mathrm{~s}$

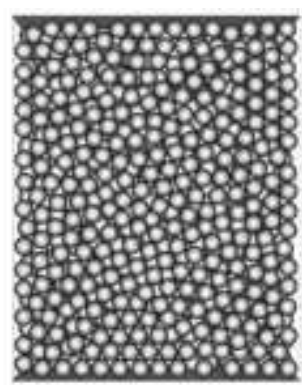

(e) $0.05 \mathrm{~s}$

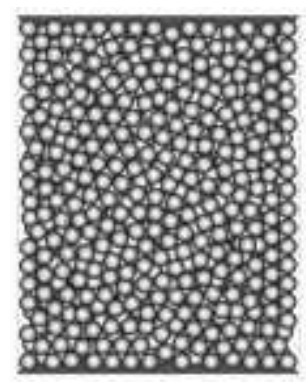

(j) $0.05 \mathrm{~s}$

Fig. 7 Snapshots from packing model for growing particle: GP1:(a) $0.0 \mathrm{~s}$; (b) $0.005 \mathrm{~s}$; (c) $0.0082 \mathrm{~s}$; (d) $0.015 \mathrm{~s}$; (e) $0.05 \mathrm{~s}$; GP2: (f) $0.0 \mathrm{~s}$; (g) $0.005 \mathrm{~s}$; (h) $0.0082 \mathrm{~s}$; (i) $0.015 \mathrm{~s}$; (j) 0.05 s.

As the simulation progresses, the size of the particles will quickly grow to fill the container. Unlike the FF model where particles fall from the top, the packing model for growing particles with full gravity fills up the container with particles from the bottom. Fig. 7(a) (e) show simulation results using the Hertz contact model at different time steps. In Fig. 7 (a) where the particles were first generated at $\mathrm{t}=0.0 \mathrm{~s}$, the particle size is $0.01 r_{p \text {,target }}$, and in Fig. 7 (b) where the size of the particles is increased and the particles begins to fall at $\mathrm{t}=0.005 \mathrm{~s}$, the particle size is $0.49 r_{p \text {,target }}$. In Fig. $7(\mathrm{c}), \mathrm{t}=0.0082 \mathrm{~s}$, the particle size reaches $0.60 r_{p, \text { target }}$, and particles are all piled up due to gravity. In Fig. $7(\mathrm{~d}), \mathrm{t}=0.015 \mathrm{~s}$, the particle size was increased to $0.88 r_{p, t a r g e t}$, and the height of the overall powder bed was increased. In Fig. 7 (e), it was found that the particle size was the same as the initial set size and it completely filled up the limited packing area. In other words, for the simulation that took about $0.13 \mathrm{~s}$ in the FF 
model, the same simulation completed in about $0.025 \mathrm{~s}$ in the growing particle model. In this model, gravity continuously affects the particles during particle generation and growth processes. This model is defined as GP1.

\subsection{Packing Model for Growing Particle with Partial Gravity (GP2)}

In the GP1 model, particles are accelerated toward the bottom of the container due to gravity. Therefore, the bottom part of the container is filled by gravity first, and then particles grow to fill the container. However, if the powder bed is inflated in this way, the growth of the powder bed will be affected by the friction between the particles and the wall, and thus, the internal particle structure may be changed. Therefore, to prevent this effect, a packing model for growing particles with a partial gradient, where gravity does not act when particles grow but acts after the size of the particles become the final set size, is used in the simulation.

Fig. 7(f) (j) shows the simulation results when the Hertz contact model was used. In Fig. 7(f), the particle size was set to $0.01 r_{p \text {,target }}$, which is the same ratio as that of the GP1 model. Likewise, in Fig. $7(\mathrm{~g})$, (h), and (i), the particles have particle size ratios of $0.49 r_{p, t a r g e t}$, $0.60 r_{p, t a r g e t}$, and $0.88 r_{p \text {,target }}$, respectively. Unlike GP1 model, gravity does not act on the particles during growing particles. Therefore, the particles do not sink but float with zero gravity. Then, the particles grow to fill the packing region, and this is defined as GP2. The final GP2 model shown in Fig. 7 does not show much difference from the final GP1 model.

\section{Data availability}

The datasets generated during the current study are available from the corresponding author on reasonable request.

\section{References}


1. $\mathrm{Fu}, \mathrm{X}$. et al. Investigation of particle packing in model pharmaceutical powders using Xray microtomography and discrete element method. Powder Technol. 167, 134-140 (2006).

2. Nan, W. et al. Jamming during particle spreding in additive manufacturing. Powder Technol. 338, 253-262 (2018).

3. Xu, W. X. \& Chen, H. S. Numerical investigation of effect of particle shape and particle size distribution on fresh cement paste microstructure via random sequential packing of dodecahedral cement particles. Comput. Struct. 114, 35-45 (2013).

4. Lee, S. G. \& Jeon, D. H. Effect of electrode compression on the wettability of lithium-ion batteries. J. Power Sources 363-369 (2014).

5. Yu, A. B. \& Standish, N. Porosity calculation of multi-component mixtures of spherical particles. Powder Technol. 52, 233-241 (1987).

6. Yu, A. B. \& Standisth, N. A study of the packing of particles with a mixture size distribution. Powder Technol. 76, 113-124 (1993).

7. Nolan, G. T. \& Kavanagh, P. E. Computer simulation of random packing of hard sphere. Powder Technol. 72, 149-155 (1992).

8. Bertei, A., Chueh, C. -C., Pharoah, J. G. \& Nicolella, C. Modified collective rearrangement sphere-assembly algorithm for random packings of nonspherical particles: Towards engineering applications. Powder Technol. 253, 311-324 (2014).

9. Muguruma, Y., Tanaka, T. \& Tsuji, Y. Numerical simulation of particulate flow with liquid bridge between particles (simulation of centrifugal tumbling granulator). Powder Technol. 109, 49-57 (2000).

10. Washino, K., Tan, H. S., Hounslow, M. J. \& Salman, A. D. A new capillary force model implemented in micro-scale CFD-DEM coupling for wet granulation. Chem. Eng. Sci. 93, 197-205 (2013).

11. Cundall, P. A. \& Strack, O. D. L. A discrete numerical model for granular assemblies. Geotechnique 29, 47-65 (1979).

12. Lee, S. \& Park, J. Standardized friction experiment for parameter determination of discrete element method and its validation using angle of repose and hopper discharge. Multiscale Sci. Eng. 1, 247-255 (2019).

13. He, Y., Evans, T. J., Yu, A. B. \& Yang, R. Y. Numerical modelling of die and unconfined compactions of wet particles. Procedia Eng. 102, 1390-1398 (2015).

14. Tangri, H., Guo, Y. \& Curtis, J. S. Packing of cylindrical particles: DEM simulations and experimental measurements. Powder Technol. 317, 72-82 (2017).

15. Deng, X. L. \& Dave, R. N. Dynamic simulation of particle packing influenced by size, aspect ratio and surface energy. Granular Matter 15, 401-415 (2013).

16. Parteli, E. J. R. et al. Attractive particle interaction forces and packing density of fine glass powders. Sci. Rep. 4, 6227 (2014).

17. Lee, Y. S., Nandwana, P. \& Zhang, W. Dynamic simulation of powder packing structure for powder bed additive manufacturing. Int. J. Adv. Manuf. Technol. 96, 1507-1520 (2018).

18. Kremmer, M. \& Favier, J. F. A method for representing boundaries in discrete element modelling-part2: Kinematics. Int. J. Numer. Meth. Eng. 51, 1423-1436 (2001).

19. Viacek, J. \& Molenda M. Effect of particles size distribution on micro- and macromechanical response of granular packings under compression. Int. J. Solids Struct. 51, 4189-4195 (2014).

20. Hearvig J. et al. On the adhesive JKR contact and rolling models for reduced particle stiffness discrete element simulations. Powder Technol. 319, 472-482 (2017).

21. Mio, H., Shimosaka, A., Shirakawa, Y. \& Hidaka, J. Optimum cell size for contact detection in the algorithm of the discrete element method. J. Chem. Eng. Japan 38(12), 969-975 (2005).

22. Sakai, M. \& Koshizuka, S. Large-scale discrete element modeling in pneumatic conveying. Chem. Eng. Sci. 64, 533-539 (2009). 
23. Sakai, M. et al. Large-scale discrete element modeling in a fluidized bed. Int. J. Numer. Meth. Fluids. 64, 1319-1335 (2010).

24. Yang, R.Y., Zou, R. P. \& Yu, A. B. Computer simulation of the packing of fine particles. Phys. Rev. E 63, 3900 (2000).

25. Dong, K. J., Zou, R. P., Yang, R. Y., Yu, A. B. \& Roach, G. DEM simulation of cake formation in sedimentation and filtration. Minerals Eng. 22, 921-930 (2009).

26. Campello, E. M. B. \& Cassares, K. R. Rapid generation of particle packs at high packing ratios for DEM simulation of granular compacts. Latin Am. J. Solids Struct. 13(1), 25-50 (2016).

27. Jerier, J. F., Richefeu, V., Imbault, D. \& Donzé, F. V. Packing spherical discrete elements for large scale simulations. Comput. Meth. Appl. Mech. Eng. 199, 1668-1676 (2010).

28. Mindlin, R. D. Compliance of elastic bodies in contact. J. Appl. Mech. 16, 259-268 (1949).

29. Johnson, K. L., Kendall, K. \& Roberts, A. D. Surface energy and the contact of elastic solids. Math. Phys. Sci. 324, 301-313 (1971).

30. Jodrey, W. S. \& Tory, E. M. Computer simulation of close random packing of equal spheres. Phys. Rev. A 32(4), 2347 (1985).

31. Jia, T., Zhang, Y. \& Chen, J. K. Simulation of granular packing of particles with different size distributions. Comput. Mater. Sci. 51, 172-180 (2012).

32. Nam, J., Lyu, J. \& Park, J. 2019. Packing structure analysis of flexible rod particles in terms of aspect ratio, bending stiffness, and surface energy. Powder Technol. 357, 232-239 (2019).

\section{Acknowledgments}

This work was supported by the National Research Foundation of Korea (NRF) funded by the Ministry of Education, Science and Technology [NRF-2018R1A2B 2004207] and the MSIT(Ministry of Science and ICT), Korea, under the Grand Information Technology Research Center support program(IITP-2020-2020-0-01612) supervised by the IITP(Institute for Information \& communications Technology Planning \& Evaluation).

\section{Author contributions}

Jinsu Nam and Jaehee Lyu wrote the main manuscript text and prepared all figures and table. All authors including corresponding author reviewed the whole manuscript.

\section{Competing interests}

The author(s) declare no competing interests. 


\section{Figure Legends}

Fig. 2 Schematic of free fall model and growing particle model: (a) Free fall method; (b) Growing particle method.

Fig. 2 Dependence of RDF between different contact model and packing model: (a) Linearspring contact model with various packing model; (b) Hertz-Mindlin contact model with various packing model; (c) Hertz-Mindlin with JKR model with various packing model.

Fig. 3 Distribution of porosity for different contact model and packing model: (a) Linear-FF model; (b) Linear-GP1 model; (c) Linear-GP2 model; (d) Hertz-FF model; (e) Hertz-GP1 model; (f) Hertz-GP2 model; (g) JKR-FF model; (h) JKR-GP1 model; (i) JKR-GP2 model.

Fig. 4 Dependence of contact number between different contact model and packing model: (a) Linear-FF model; (b) Linear-GP1 model; (c) Linear-GP2 model; (d) Hertz-FF model; (e) Hertz-GP1 model; (f) Hertz-GP2 model; (g) JKR-FF model; (h) JKR-GP1 model; (i) JKR-GP2 model.

Fig. 5 Comparison to simulation running time between packing models.

Fig. 6 Snapshots for free fall particle packing: (a) $0.0 \mathrm{~s}$; (b) $0.01 \mathrm{~s}$; (c) $0.02 \mathrm{~s}$; (d) $0.05 \mathrm{~s}$; (e) $0.15 \mathrm{~s}$.

Fig. 7 Snapshots from packing model for growing particle: GP1:(a) $0.0 \mathrm{~s}$; (b) $0.005 \mathrm{~s}$; (c) 0.0082 s; (d) $0.015 \mathrm{~s}$; (e) $0.05 \mathrm{~s}$; GP2: (f) $0.0 \mathrm{~s}$; (g) $0.005 \mathrm{~s}$; (h) $0.0082 \mathrm{~s}$; (i) $0.015 \mathrm{~s}$; (j) $0.05 \mathrm{~s}$. 
Tables

Table 2 Material properties

\begin{tabular}{lc}
\hline Particle parameter & Value \\
\hline Particle size, $r_{p}$ & $25 \mu \mathrm{m}$ \\
\hline Particle density, $\rho$ & $2500 \mathrm{~kg} / \mathrm{m}^{3}$ \\
\hline Total number of particles & 5000 \\
\hline Young's modulus, $E$ & $6.3 \times 10^{9} \mathrm{~Pa}$ \\
\hline Poisson's ratio, $v$ & 0.24 \\
\hline Coefficient of restitution, $\varepsilon_{p}$ & 0.95 \\
\hline Friction coefficient (particle to particle), $\mu_{p}$ & 0.1 \\
\hline Friction coefficient (particle to wall), $\mu_{w}$ & 0.0 \\
\hline Surface energy (only for JKR model), $\gamma$ & $0.0199 \mathrm{~N} / \mathrm{m}^{2}$ \\
\hline Wall parameter & \\
\hline Upper plate velocity & $-2 \mathrm{~mm} / \mathrm{s}$ \\
\hline Compaction ratio & 0.943 \\
\hline
\end{tabular}


Figures

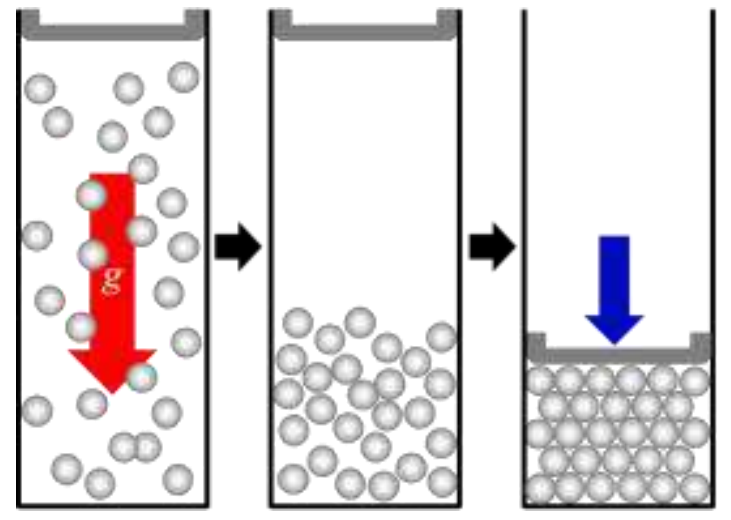

(a)

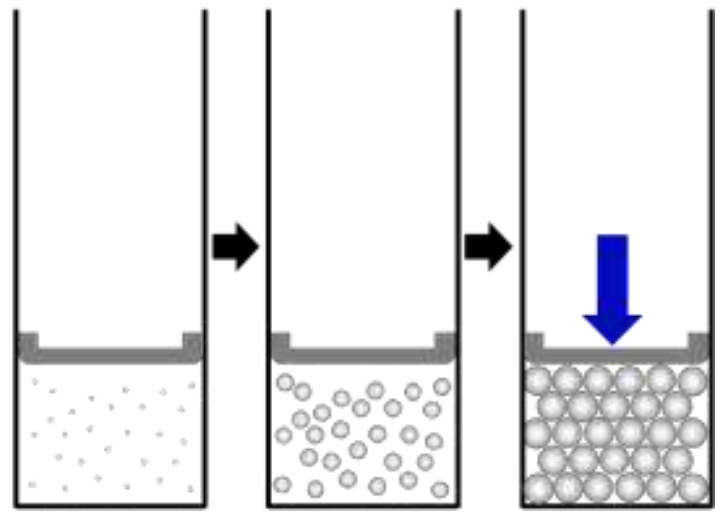

(b)

Fig. 3

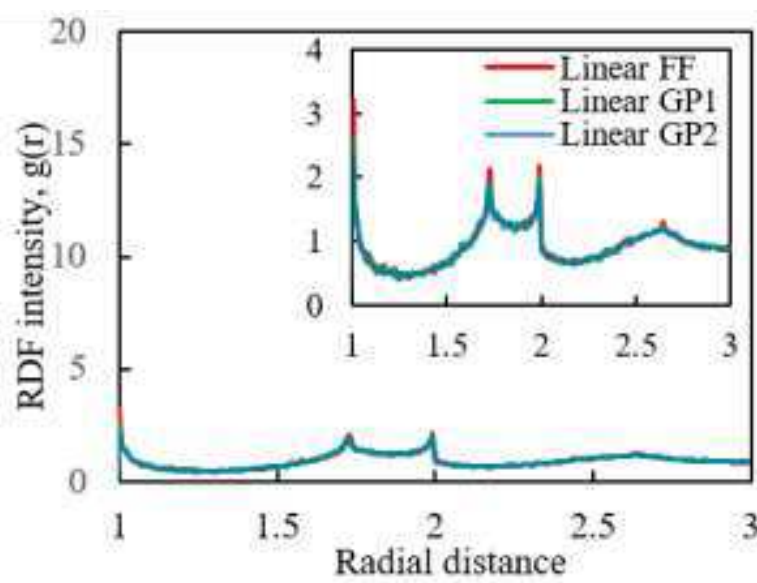

(a)

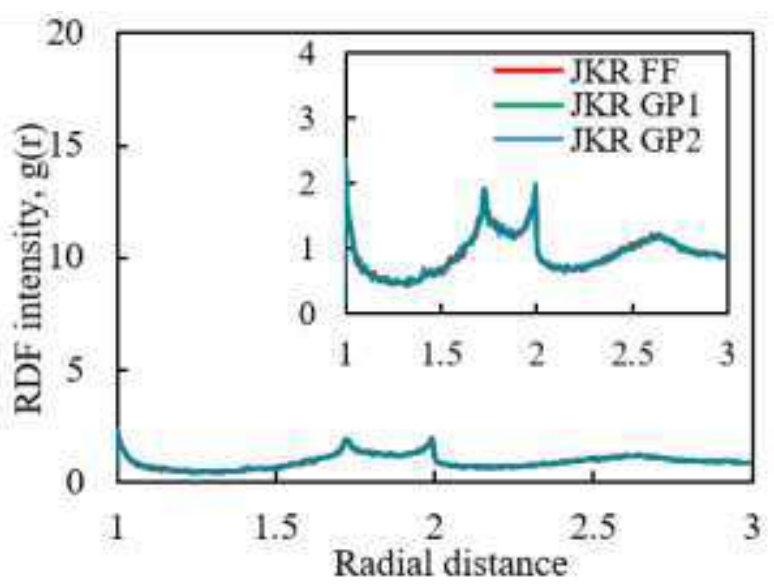

(c)

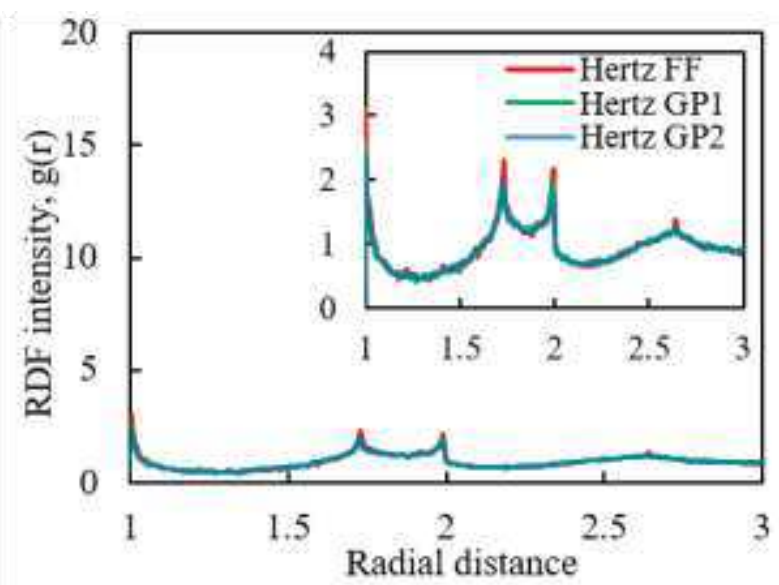

(b)

Fig. 2 


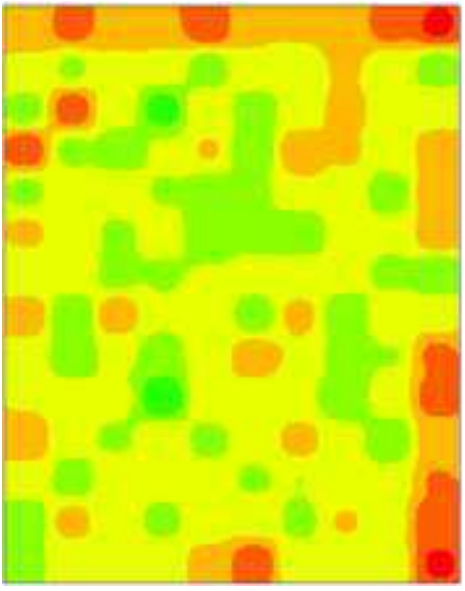

(a) Linear-FF model;

Linear-GP2 model;

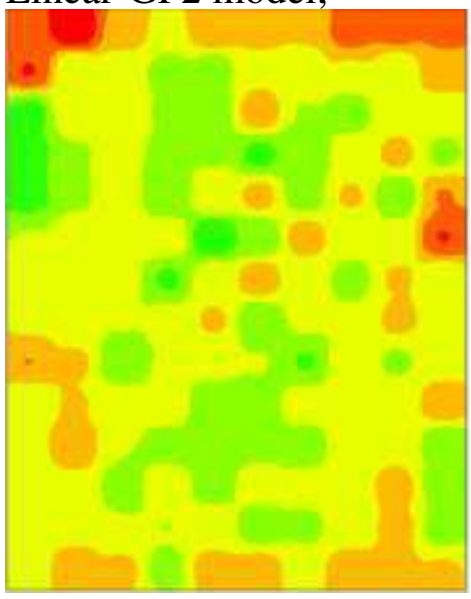

(d) Hertz-FF model;

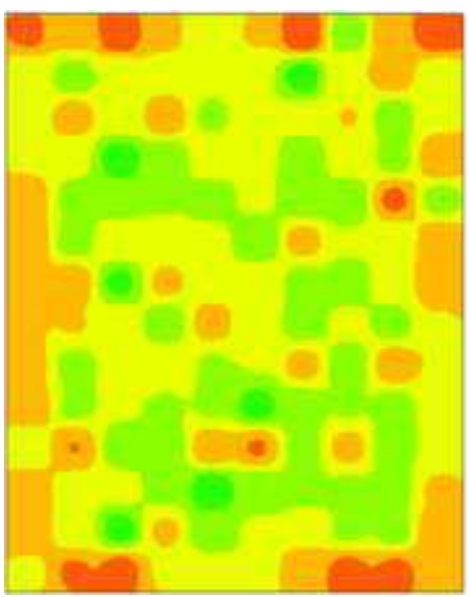

(g) JKR-FF model;
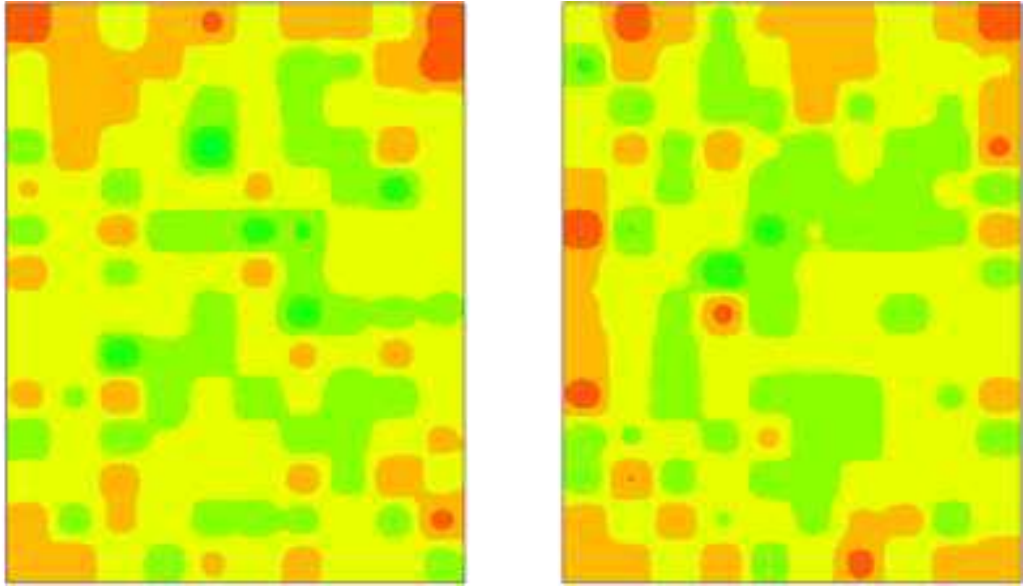

(b) Linear-GP1 model;

(c)

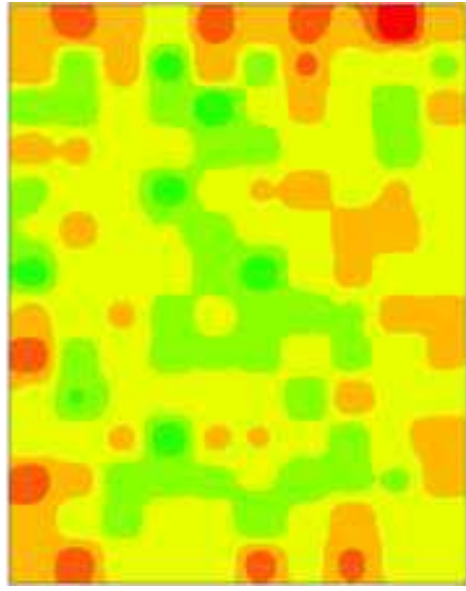

(e) Hertz-GP1 model;

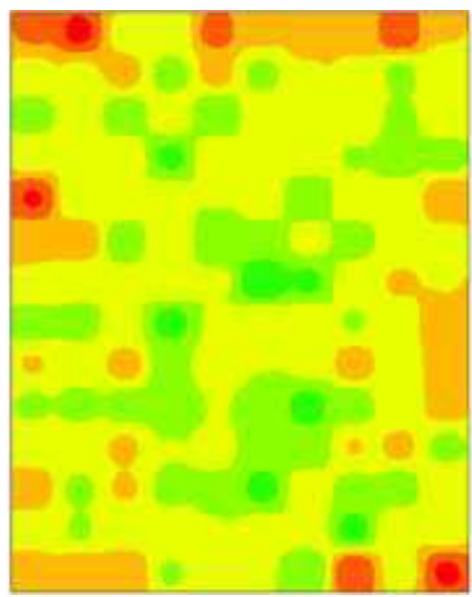

(h) JKR-GP1 model;

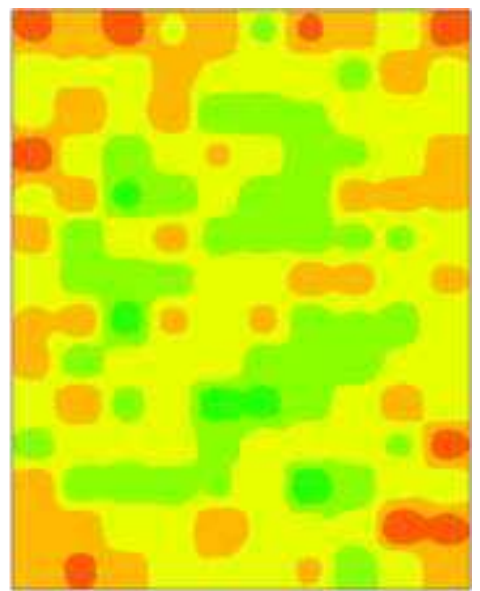

(f) Hertz-GP2 model;

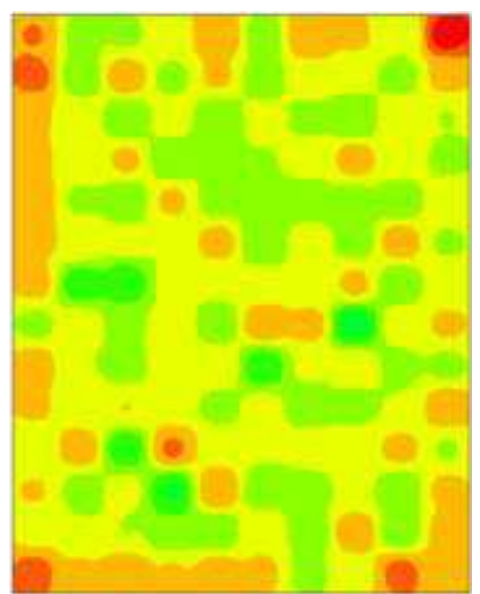

(i) JKR-GP2 model;

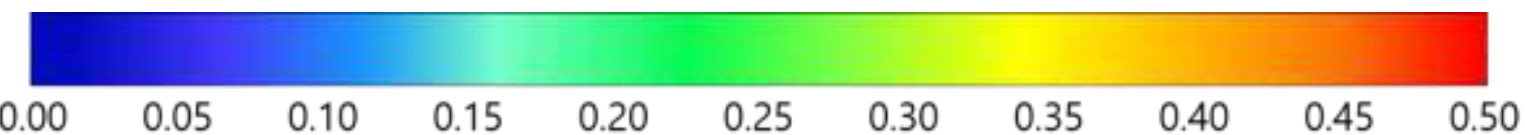

Fig. 3 


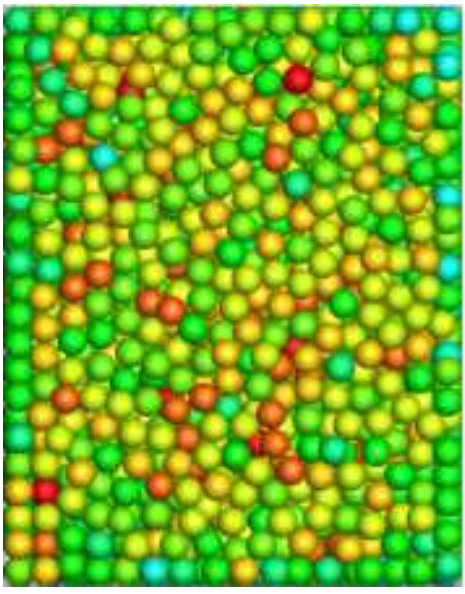

(a) Linear-FF model; model;

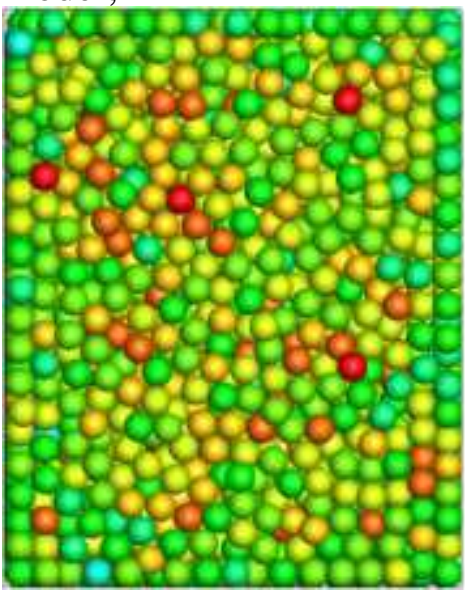

(d) Hertz-FF model;

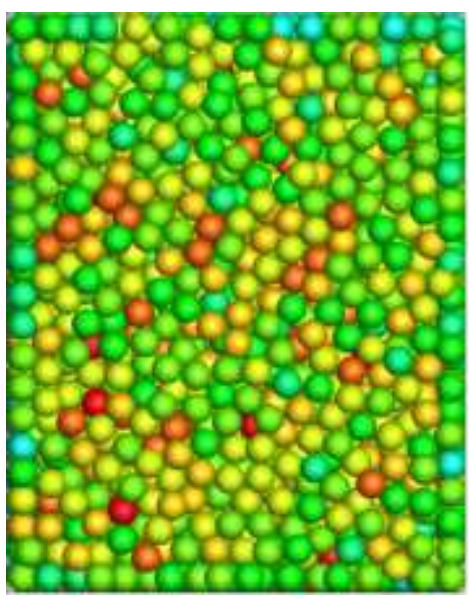

(g) JKR-FF model;

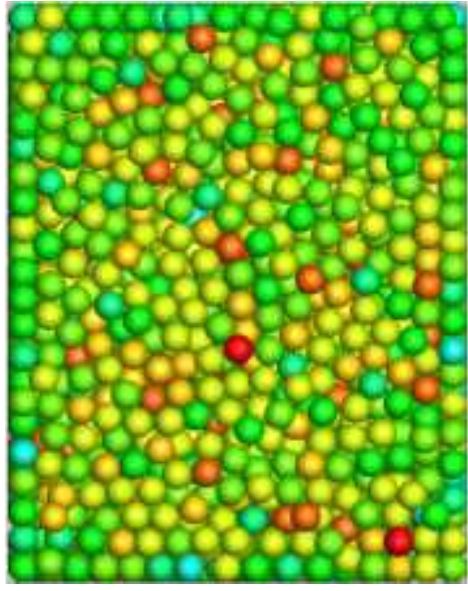

(b) Linear-GP1 model;

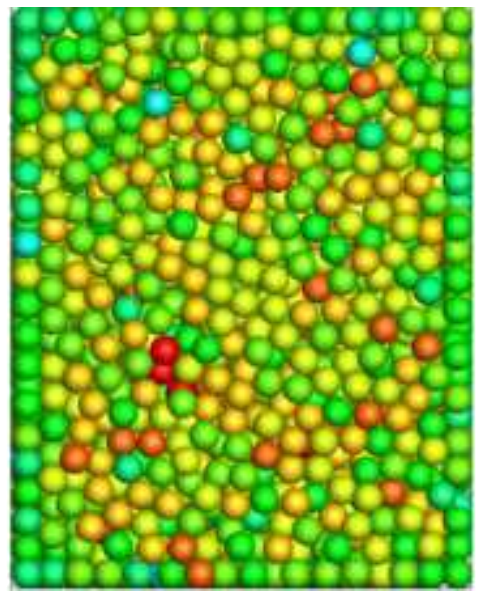

(e) Hertz-GP1 model;

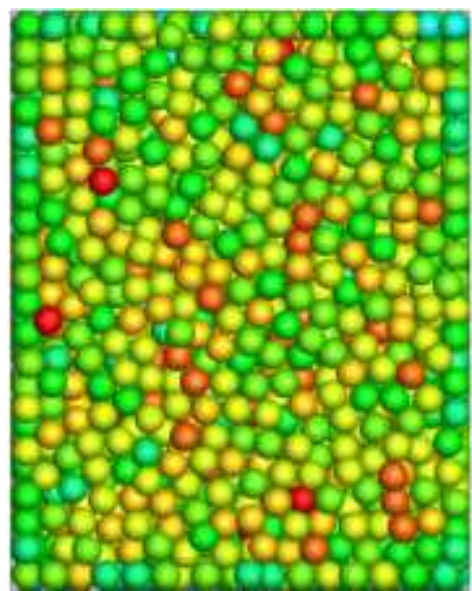

(h) JKR-GP1 model;

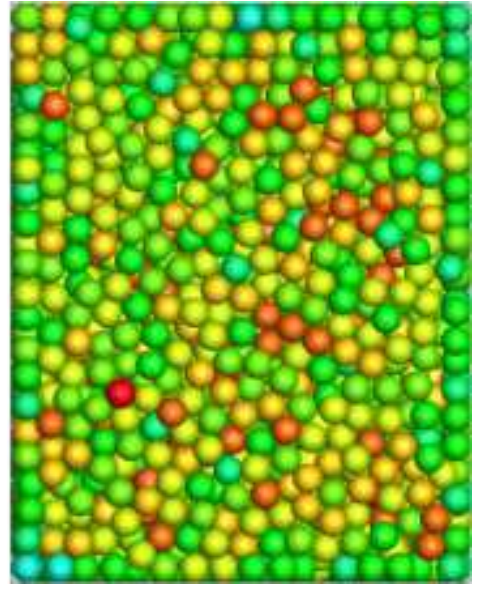

(c) Linear-GP2

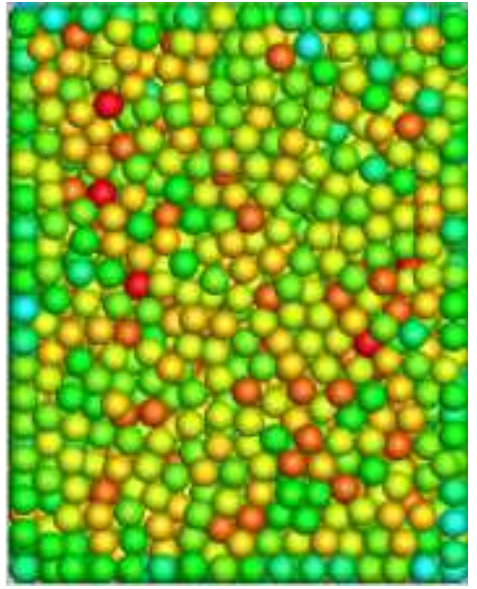

(f) Hertz-GP2 model;

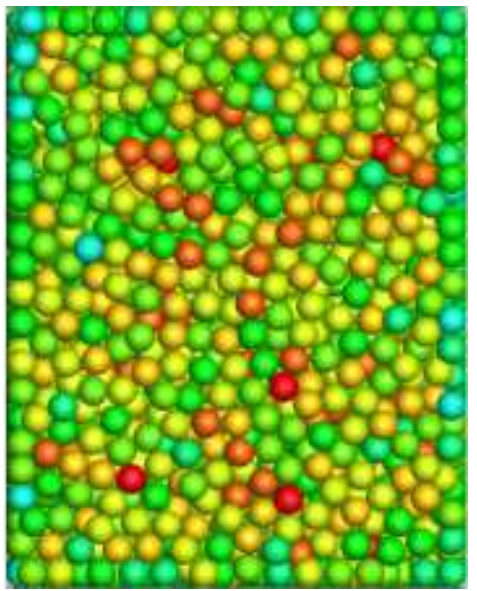

(i) JKR-GP2 model

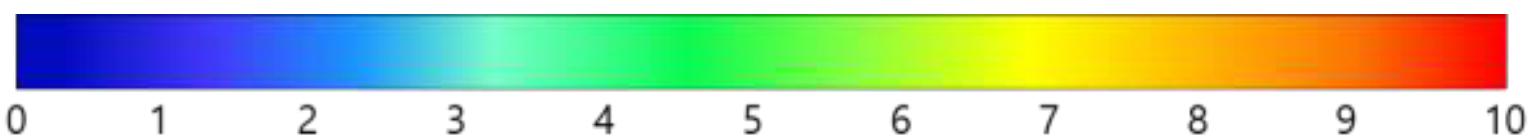

Fig. 4 


\section{Fig. 5}

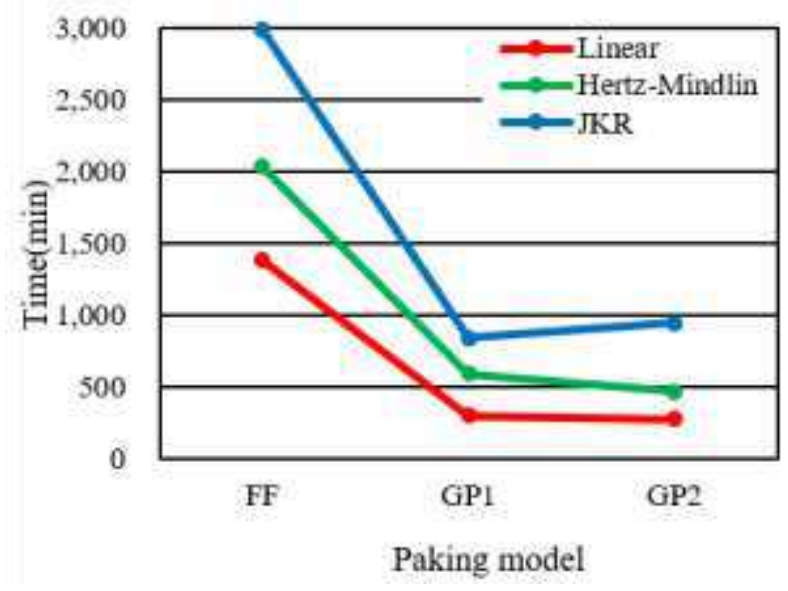

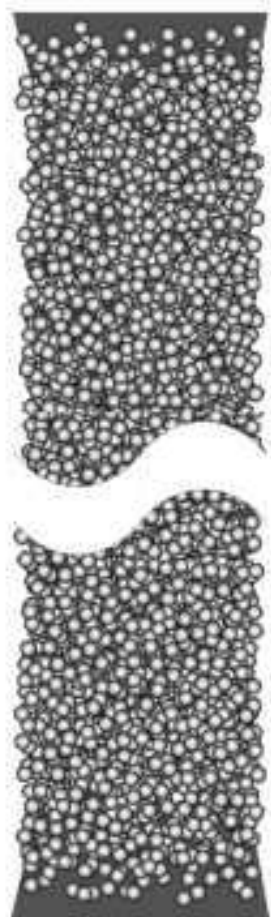

(a) $0.0 \mathrm{~s}$

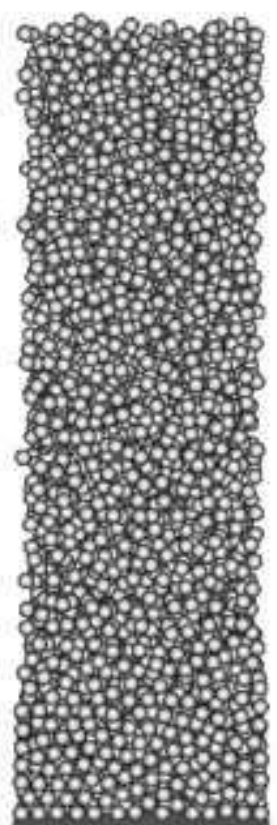

(b) $0.01 \mathrm{~s}$

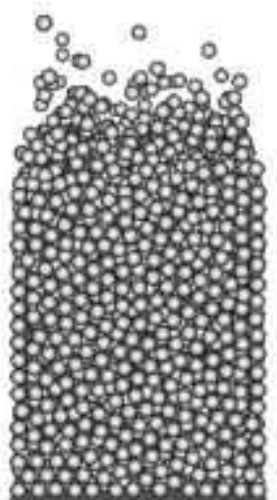

(c) $0.02 \mathrm{~s}$

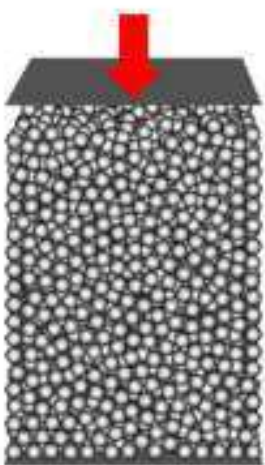

(d) $0.05 \mathrm{~s}$

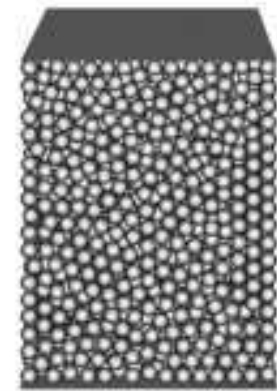

(e) $0.15 \mathrm{~s}$

Fig. 6 


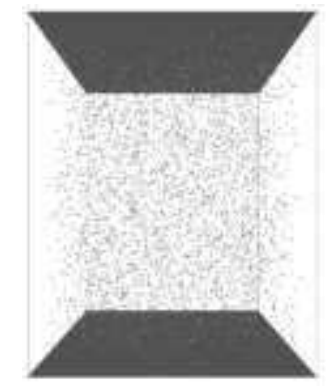

GP1: (a) $0.0 \mathrm{~s}$

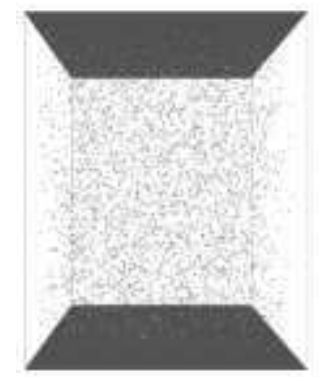

GP2: (f) $0.0 \mathrm{~s}$

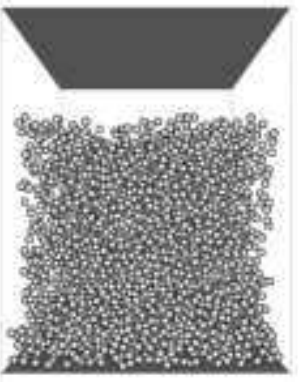

(b) $0.005 \mathrm{~s}$

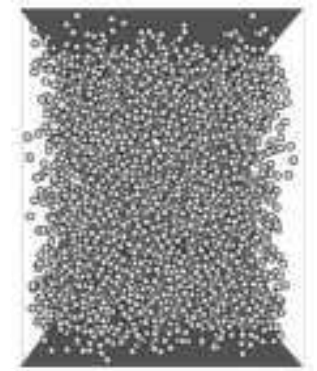

(g) $0.005 \mathrm{~s}$

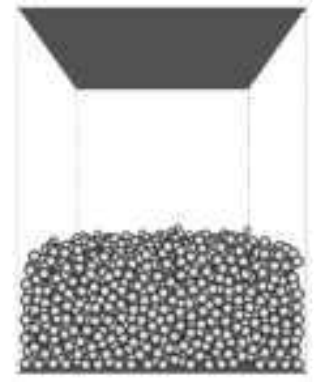

(c) $0.0082 \mathrm{~s}$

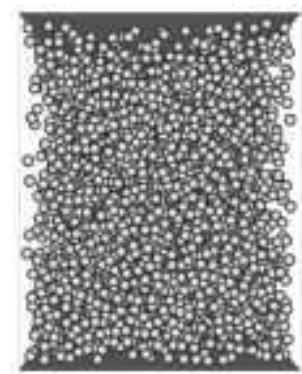

(h) $0.0082 \mathrm{~s}$

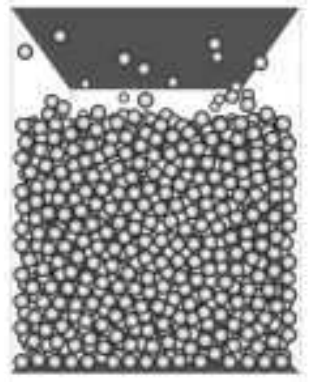

(d) $0.015 \mathrm{~s}$

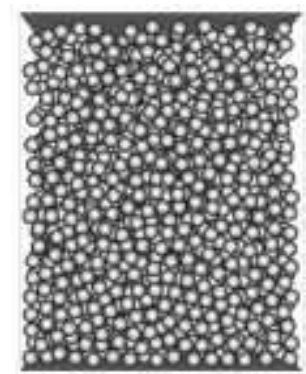

(i) $0.015 \mathrm{~s}$

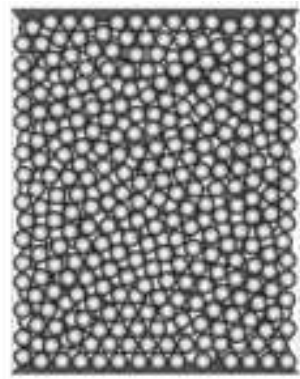

(e) $0.05 \mathrm{~s}$

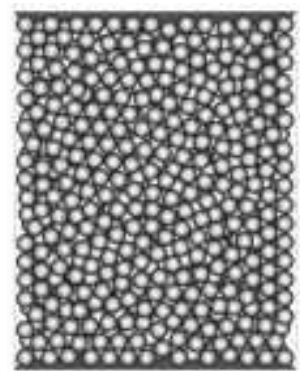

(j) $0.05 \mathrm{~s}$

Fig. 7 